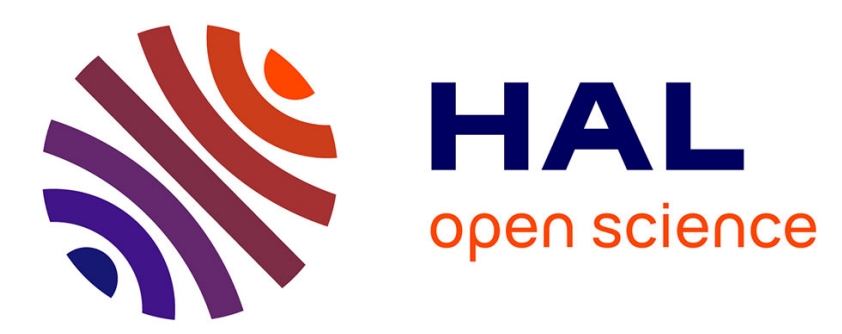

\title{
Geometric desingularization of a cusp singularity in slow-fast systems with applications to Zeeman's examples
}

\author{
H. Broer, Tasso J. Kaper, Maciej Krupa
}

\section{- To cite this version:}

H. Broer, Tasso J. Kaper, Maciej Krupa. Geometric desingularization of a cusp singularity in slowfast systems with applications to Zeeman's examples. Journal of Dynamics and Differential Equations, 2013. hal-00846008

\author{
HAL Id: hal-00846008 \\ https://hal.inria.fr/hal-00846008
}

Submitted on 18 Jul 2013

HAL is a multi-disciplinary open access archive for the deposit and dissemination of scientific research documents, whether they are published or not. The documents may come from teaching and research institutions in France or abroad, or from public or private research centers.
L'archive ouverte pluridisciplinaire HAL, est destinée au dépôt et à la diffusion de documents scientifiques de niveau recherche, publiés ou non, émanant des établissements d'enseignement et de recherche français ou étrangers, des laboratoires publics ou privés. 


\title{
Geometric desingularization of a cusp singularity in slow-fast systems with applications to Zeeman's examples
}

\author{
Henk W. Broer, Tasso J. Kaper, Martin Krupa \\ original May 13, 2008; revised November 25, 2010
}

\begin{abstract}
The cusp singularity — a point at which two curves of fold points meet - is a prototypical example in Takens' classification of singularities in constrained equations, which also includes folds, folded saddles, folded nodes, among others. In this article, we study cusp singularities in singularly perturbed systems for sufficiently small values of the perturbation parameter, in the regime in which these systems exhibit fast and slow dynamics. Our main result is an analysis of the cusp point using the method of geometric desingularization, also known as the blow-up method, from the field of geometric singular perturbation theory. Our analysis of the cusp singularity was inspired by the nerve impulse example of Zeeman, and we also apply our main theorem to it. Finally, a brief review of geometric singular perturbation theory for the two elementary singularities from the Takens' classification occurring for the nerve impulse example — folds and folded saddles — is included to make this article self-contained.
\end{abstract}

This article is dedicated to Freddy Dumortier on the occasion of his sixtieth birthday.

\section{Contents}

1 Introduction $\quad 2$

2 Analysis of Zeeman's models as constrained equations $\quad 4$

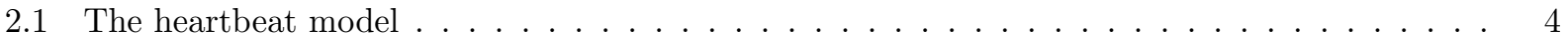

2.2 The nerve impulse model $\ldots \ldots \ldots \ldots \ldots \ldots \ldots$

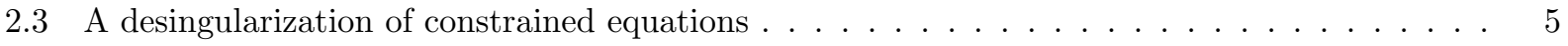

2.4 Folds, folded saddles, and cusps in constrained equations with two parameters . . . . . . . 7

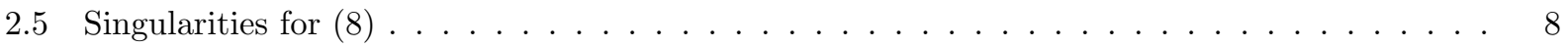

2.6 Singular global return for Zeeman's equation $(8) \ldots \ldots \ldots \ldots \ldots$

3 Singularly perturbed cusps - statement of the main theorem $\quad 14$

4 Analysis of a singularly perturbed cusp by means of geometric desingularization 16

4.1 Dynamics in the entry chart $K_{\mathrm{en}} \ldots \ldots \ldots \ldots \ldots \ldots \ldots$

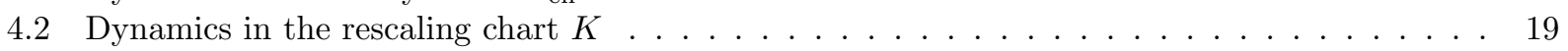

4.3 Dynamics in the rescaling charts $K_{-}$and $K_{+} \ldots \ldots \ldots \ldots \ldots \ldots \ldots \ldots$

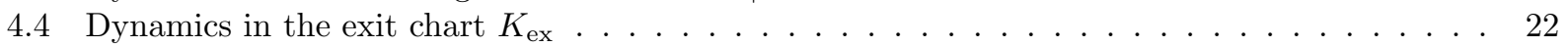

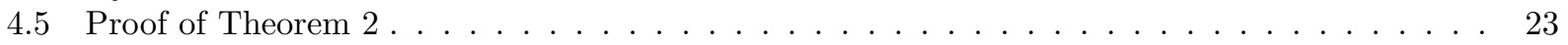

5 Analysis for initial conditions lying below a neighborhood of the cusp point 24

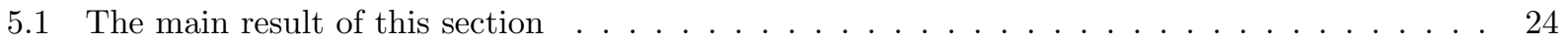

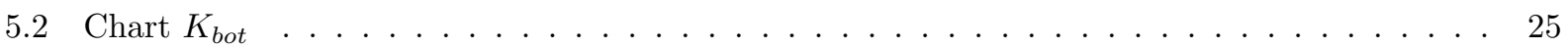

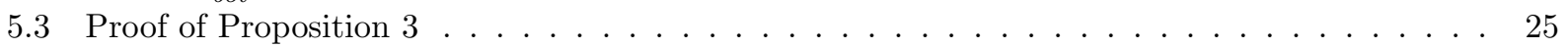

6 Application to Zeeman's examples 2

6.1 The results for the heartbeat model $(4)$ with $\varepsilon>0 \ldots \ldots \ldots \ldots \ldots \ldots$

6.2 The results for the nerve impulse model $(7)$ with $\varepsilon>0 \ldots \ldots \ldots \ldots \ldots$ 
B Folds and folded saddles for $\varepsilon>0$ - review 29

B.1 Simple folds . . . . . . . . . . . . . . . . . . . . . . . . 29

B.2 Simple folds in problems with two slow dimensions $\ldots \ldots \ldots \ldots \ldots \ldots \ldots$

B.3 Folded saddles . . . . . . . . . . . . . . . . . . . . . . . . . . . 32

\section{Introduction}

Consider a singularly perturbed system of the form

$$
\begin{aligned}
\dot{x} & =f(x, y) \\
\varepsilon \dot{y} & =g(x, y), \quad x \in \mathbb{R}^{k}, y \in \mathbb{R}^{m},
\end{aligned}
$$

where $\varepsilon>0$ is small. The small parameter $\varepsilon$ measures the relative rates of change of $x$ and $y$, and one sees that the smaller $\varepsilon$ is the faster $y$ evolves relative to $x$, as long as $g(x, y) \neq 0$. In the limit $\varepsilon=0$, system (1) reduces to the differential-algebraic system

$$
\begin{aligned}
& \dot{x}=f(x, y) \\
& 0=g(x, y), \quad x \in \mathbb{R}^{k}, y \in \mathbb{R}^{m} .
\end{aligned}
$$

in which $x$ evolves slowly while the fast variable $y$ adjusts instantaneously to satisfy the constraint that $g$ vanishes. System (2) provides a leading order approximation to (1) and is known in singular perturbation theory as either the constrained equation [14] or the reduced system [8]. Another approximation used in singular perturbation theory, namely the fast equation, is obtained as follows. Rescaling time in (1) by letting $t=\varepsilon \tau$, we obtain the equation

$$
\begin{aligned}
& x^{\prime}=\varepsilon f(x, y) \\
& y^{\prime}=g(x, y), \quad x \in \mathbb{R}^{k}, y \in \mathbb{R}^{m},
\end{aligned}
$$

where ' denotes the derivative with respect to $\tau$. Setting $\varepsilon=0$, we obtain the so-called layer problem [10], in which the systems is reduced to $m$ ODEs for the fast variable $y$ which depend on the slow variable $x$ as a parameter.

Constrained equations are equivalent to equations without a constraint at points where $D_{y} g$ is invertible. Near such points, the constraint $g(x, y)=0$ can be eliminated by solving for $y$ as a function of $x$. By contrast, points where $D_{y} g$ is not invertible are called singularities. The set $S_{0}=\{g(x, y)=0\}$ is referred to as the constraining manifold in [14]; it is the phase space of (2). In the literature on singular perturbation theory, $S_{0}$ is also known as the critical manifold [8], or slow manifold. Note that $S_{0}$ is the set of equilibria for (3) with $\varepsilon=0$. Moreover, transverse to $S_{0}$, the fast directions are understood as the infinitely fast foliation in [14], and as the fast fibers in the Fenichel theory, see [8]. The setup of constrained equations is schematically illustrated in Figure 1.

One can define a hybrid system using the dynamics of (2) and (3) in the following way. A point away from $S_{0}$ moves infinitely fast along a stable fast fiber, following the dynamics of (3) with $\varepsilon=0$, until it reaches a stable branch of $S_{0}$. On $S_{0}$, the dynamics switches to (2). If the corresponding solution reaches a singularity or a bifurcation point (loss of stability of $S_{0}$ ), then the dynamics switches back to (3). The relation between the dynamics of (1) with $0<\varepsilon \ll 1$ and the hybrid system described above is well understood away from singularities by Fenichel theory, but there are still many unanswered questions regarding the flow near singularities.

Takens [14] developed a local theory of constrained equations using singularity theory. He classified a number of singularities and found normal forms determining equivalence classes under topological conjugacy. The motivation for his analysis came from articles by Zeeman [19] and by Thom [16, 17, 18]. A challenging problem is to extend the analysis of [14] to the context of (1) with $0<\varepsilon \ll 1$. Such an extension has been carried out for all known singularities in the cases of problems with dimension $k \leq 2$ [1, 10, 12], with the exception of the cusp singularity. In this article, the main result is a complete analysis of the cusp singularity 


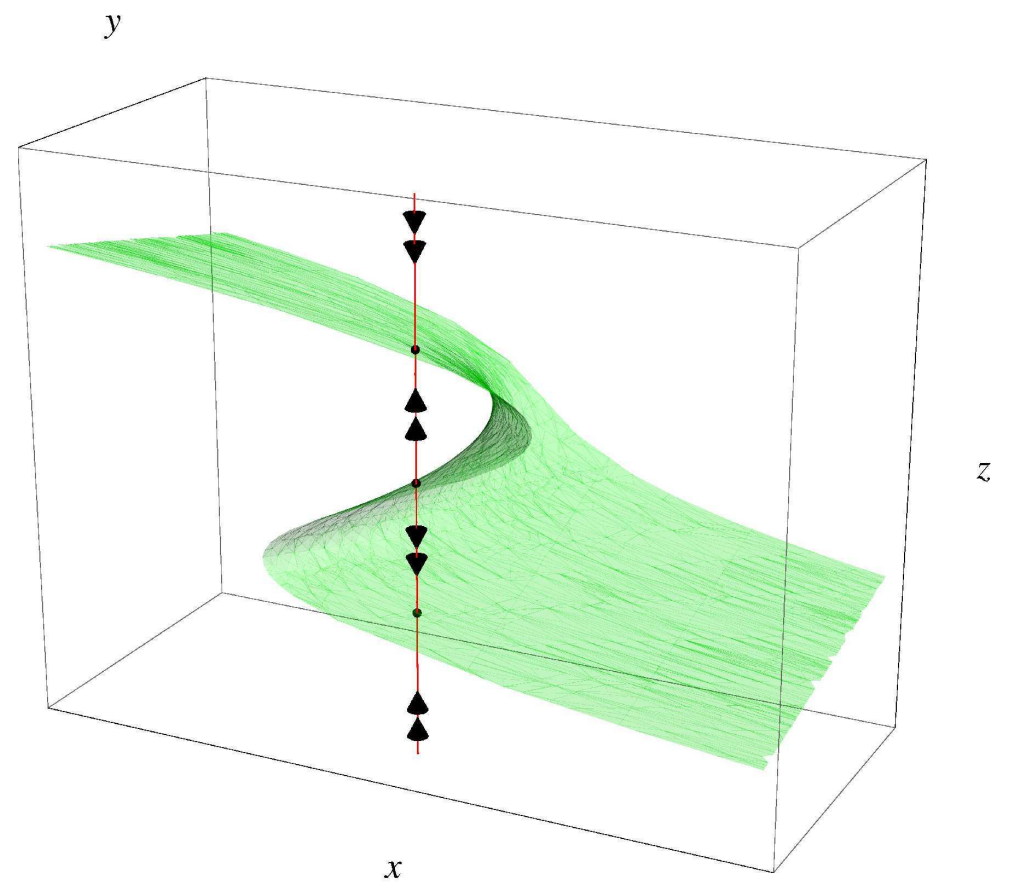

Figure 1: The cusp singularity and the infinitely fast foliation

for systems (1) with $0<\varepsilon \ll 1$, and therefore we complete the study of generic singularities in dimensions $k \leq 2$ for problems $(1)$.

In addition to presenting this main result about the cusp singularity, we also apply it to obtain a complete description of the dynamics of the toy nerve impulse model of Zeeman [19], in which a cusp singularity plays a central role. We demonstrate that there exist solutions exhibiting smooth returns, and we think that a quantitative analysis of when this phenomenon of smooth returns arises will be useful for understanding the dynamics of other problems exhibiting cusps. Moreover, this model also has a folded saddle singularity, and hence we will also employ known results on folds and folded saddles from [12] and [13]. Finally, for completeness, we also present the analysis of the heartbeat model introduced in [19] (a two variable toy model of a heart cell), which is a simple relaxation oscillator. We note that more realistic Hodgkin-Huxleylike models of specific neurons and more realistic heartbeat models have been developed and analyzed. For some of these (see for example [15]), folded nodes and folded saddle nodes are the central singularities, and the presence of two or more of these singularities leads to interesting recurrent dynamics.

This article is organized as follows. In Section 2, we present the analysis of Zeeman's models in the setting of constrained equations. In Section 3, we state our main result about cusps (Theorem 2). This main theorem, which treats almost all orbits that enter the neighborhood of the cusp, is then proven in Section 4. Some initial conditions enter the cusp region from below a small neighborhood of the cusp point, and we study their dynamics in Section 5. Finally, in Section 6, we prove theorems about the dynamics of the heartbeat model (Theorem 3) and about the dynamics of the nerve impulse model (Theorem 4). 


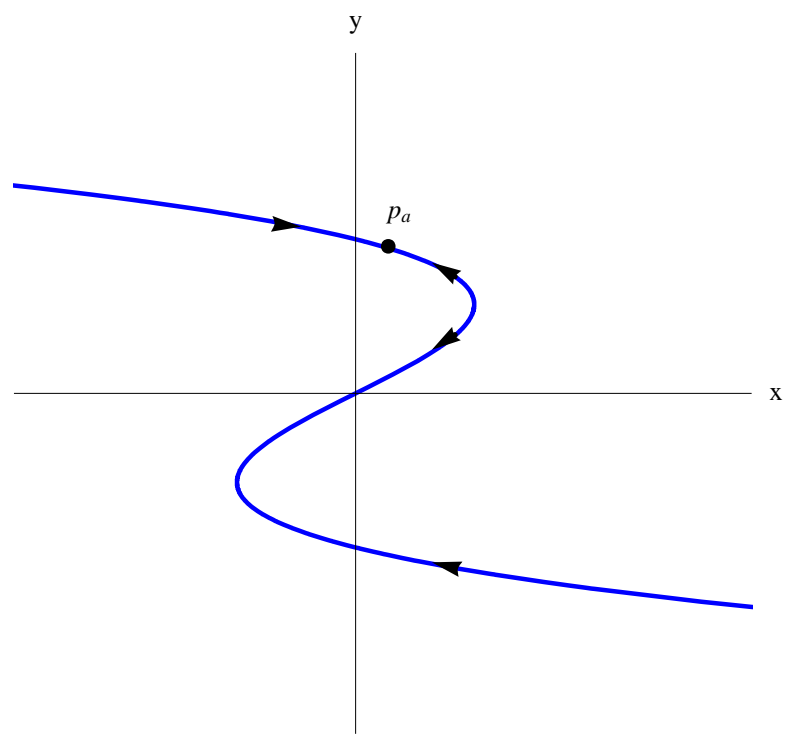

Figure 2: Dynamics of the reduced equation (6), with $p_{a}$ denoting the stable equilibrium.

\section{Analysis of Zeeman's models as constrained equations}

\subsection{The heartbeat model}

In [19], Zeeman introduced the following elementary model:

$$
\begin{aligned}
\dot{x} & =y-y_{0} \\
\varepsilon \dot{y} & =-\left(y^{3}-y+x\right),
\end{aligned}
$$

where $y_{0}$ is a constant greater than $1 / \sqrt{3}$. It is an excitable dynamical system, [9], and at the time it was a simple caricature (now surpassed by more realistic models) of electrical activity in heart muscle. Zeeman constructed the model so that the stable equilibrium has a relatively small basin of attraction. A suitable perturbation (small in size) makes the system jump to a different slow manifold, but eventually the trajectory then returns to the stable equilibrium via a jump return, i.e. a fast transition between the two different stable attracting slow manifolds.

Setting $\varepsilon=0$ in (4), we obtain the following constrained equations:

$$
\begin{aligned}
& \dot{x}=y-y_{0} \\
& 0=-\left(y^{3}-y+x\right) .
\end{aligned}
$$

The critical (constraining) manifold $S_{0}$ is defined by the condition $y^{3}-y+x=0$. This curve has two fold points at $y= \pm 1 / \sqrt{3}$. We can eliminate the constraint using the fact that $S_{0}$ is a graph of $x$ as a function of $y$, namely $x=-y^{3}+y$. Differentiating this relationship with respect to $t$, we get $\dot{x}=\left(1-3 y^{2}\right) \dot{y}$. Substituting into (5) and dividing through by the factor $1-3 y^{2}$, we get the reduced equation

$$
\dot{y}=\frac{1}{1-3 y^{2}}\left(y-y_{0}\right) \text {. }
$$

Equation (6) has singularities at the fold points $y= \pm 1 / \sqrt{3}$, and the flow is as shown in Figure 2.1.

Analysis of equation (4) with $0<\varepsilon \ll 1$ is presented in Section 6 . 


\subsection{The nerve impulse model}

Zeeman [19] also introduced the following elementary model for transmembrane voltage in a nerve cell:

$$
\begin{aligned}
\dot{x} & =-1-y \\
\dot{y} & =-2(y+z) \\
\varepsilon \dot{z} & =-\left(x+y z+z^{3}\right),
\end{aligned}
$$

as a caricature of the Hodgkin-Huxley equations. An important feature of (7) is the possibility of smooth return, i.e. after the jump away from the equilibrium the trajectory returns staying all the time on the slow manifold. In this section we study the constrained version of (7),

$$
\begin{aligned}
& \dot{x}=-1-y \\
& \dot{y}=-2(y+z) \\
& 0=x+y z+z^{3} .
\end{aligned}
$$

In Section 6, we extend our analysis to equation (7).

The central feature of the constraint surface $x+y z+z^{3}=0$ is the cusp singularity at the origin, from which two fold lines emanate. It turns out that one of the fold lines also contains a folded saddle singularity. In the next two sections (Sections 2.3 and 2.4), we review the results and the analysis of these singularities in the setting of constrained equations. Subsequently, in Sections 2.5 and 2.6, we return to (8) and analyze its singularities in complete detail.

\subsection{A desingularization of constrained equations}

We consider constrained equations of the general form

$$
\begin{aligned}
& \dot{x}=f_{1}(x, y, z) \\
& \dot{y}=f_{2}(x, y, z) \\
& 0=g(x, y, z) .
\end{aligned}
$$

Assumptions: we consider the flow of $(9)$ near a point $\left(x_{0}, y_{0}, z_{0}\right)$ satisfying the defining conditions

$$
\begin{aligned}
& g\left(x_{0}, y_{0}, z_{0}\right)=0 \\
& g_{z}\left(x_{0}, y_{0}, z_{0}\right)=0
\end{aligned}
$$

Further, we need a non-degeneracy condition, denoted by (A),

$$
\text { (A) } \quad g_{(x, y)}\left(x_{0}, y_{0}, z_{0}\right) \neq 0 .
$$

The following result is well known, see for example $[12,14]$ (we include the proof for completeness).

Lemma 1 Assume (10) and (A). Then, the constrained equation (9) can be written in the form

$$
\begin{aligned}
\dot{y} & =f_{2}(\varphi(y, z), y, z) \\
-g_{z}(\varphi(y, z), y, z) \dot{z} & =f(\varphi(y, z), y, z) \cdot g_{(x, y)}(\varphi(y, z), y, z) .
\end{aligned}
$$

with $x=\varphi(y, z)$ solving $g(x, y, z)=0$.

Proof Note that (A) implies that either $g_{x}\left(x_{0}, y_{0}, z_{0}\right) \neq 0, g_{y}\left(x_{0}, y_{0}, z_{0}\right) \neq 0$, or both are nonzero. We assume without loss of generality that $g_{x}\left(x_{0}, y_{0}, z_{0}\right) \neq 0$. Since we can solve the constraint for $x$, the idea is to use the variables $(y, z)$ to represent (9). (One may also solve for $y$ as a function of $x$ and $z$ if $g_{y} \neq 0$.) The solution has the form

$$
x=\varphi(y, z),
$$


with

$$
g(\varphi(y, z), y, z)=0 .
$$

Differentiating (13) with respect to $t$, we derive the expression

$$
\dot{x}=\varphi_{y} \dot{y}+\varphi_{z} \dot{z}
$$

Using implicit differentiation, we obtain

$$
\varphi_{z}=-\frac{g_{z}}{g_{x}} ; \quad \varphi_{y}=-\frac{g_{y}}{g_{x}}
$$

which implies

$$
-\frac{g_{z}}{g_{x}} \dot{z}=f_{1}+\frac{g_{y}}{g_{x}} f_{2}
$$

or

$$
-g_{z} \dot{z}=g_{(x, y)} \cdot f \text {. }
$$

The result (12) follows.

Note that (12) has singularities along the fold line. Using Cramer's rule to solve for $(\dot{y}, \dot{z})$, we can rewrite (12) as follows:

$$
\begin{aligned}
& \dot{y}=\left(-\frac{1}{g_{z}}\right)\left(-g_{z} f_{2}\right) \\
& \dot{z}=\left(-\frac{1}{g_{z}}\right) g_{(x, y)} \cdot f
\end{aligned}
$$

Cancelling the factor $\left(-1 / g_{z}\right)$, we obtain the desingularized equation

$$
\begin{aligned}
& \dot{y}=-g_{z} f_{2} \\
& \dot{z}=g_{(x, y)} \cdot f
\end{aligned}
$$

with $x$ determined by the equation $x=\varphi(y, z)$. Away from the fold line, trajectories of (12) and (15) differ by time parametrization only. The direction of time is reversed in the region where $-g_{z}<0$.

We introduce additional non-degeneracy conditions

$$
g_{(x, y)}\left(x_{0}, y_{0}, z_{0}\right) \cdot f\left(x_{0}, y_{0}, z_{0}\right) \neq 0 .
$$

and

$$
\text { (C) } \quad g_{z z}\left(x_{0}, y_{0}, z_{0}\right) \neq 0
$$

\section{Classification of singular points}

Simple fold points are the points for which both (B) and (C) hold.

Folded equilibria are points for which (B) fails but (C) holds. Folded equilibria occur generically at isolated points on a fold line. Additional non-degeneracy conditions may apply, but we do not discuss them here. Cusp points are points for which (B) holds but (C) fails. Cusp points occur generically at isolated points of a fold line where two fold lines meet. For a non-degenerate cusp point, the non-degeneracy condition

$$
\text { (D) } \quad g_{z z z}\left(x_{0}, y_{0}, z_{0}\right) \neq 0
$$

is needed. 


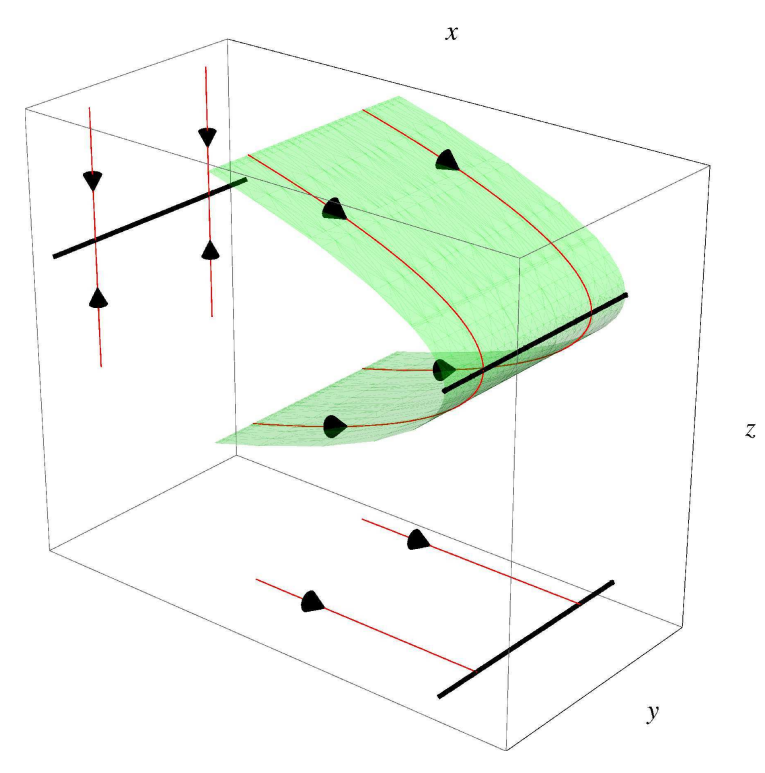

Figure 3: Simple fold point, normal form (16) with plus sign. Corresponds to Figure 3, Case 5 in [14].

\subsection{Folds, folded saddles, and cusps in constrained equations with two param- eters}

Takens [14] found simple normal forms for a number of constrained equations and proved a result asserting topological conjugacy between singularities in general position and the corresponding normal forms [14, Theorem 5.1, p. 178]. In this section, we review the normal forms given in [14] for the three cases relevant to the nerve impulse model (7): folds, folded saddles, and cusps.

The normal form for a fold is as follows:

$$
\begin{aligned}
\dot{x} & = \pm 1 \\
\dot{y} & =0 \\
0 & =-\left(z^{2}+x\right) .
\end{aligned}
$$

(The normal form for a fold with one slow variable is obtained by eliminating the $y$ variable). Note that both (B) and (C) are satisfied for (16). The dynamics can be understood using the method outlined in Section 2.3. The phase portrait, corresponding to the plus sign in the $x$ equation, is shown in Figure 3.

The normal form for a folded saddle is as follows:

$$
\begin{aligned}
& \dot{x}=-y \\
& \dot{y}=1 \\
& 0=z^{2}+x .
\end{aligned}
$$

Note that condition (B) fails but (C) holds. The dynamics can be understood using the method outlined in Section 2.3. The phase portrait is shown in Figure 4.

The normal form for a cusp is as follows:

$$
\begin{aligned}
& \dot{x}=1 \\
& \dot{y}=0 \\
& 0= \pm\left(z^{3}+y z+x\right) .
\end{aligned}
$$

The choice of the sign in (18) determines the stability of the sheets of $g(x, y, z)=0$ for the dynamics of the infinitely fast foliation, namely the sheets for which $g_{z}<0$ are defined as stable and the sheets for 


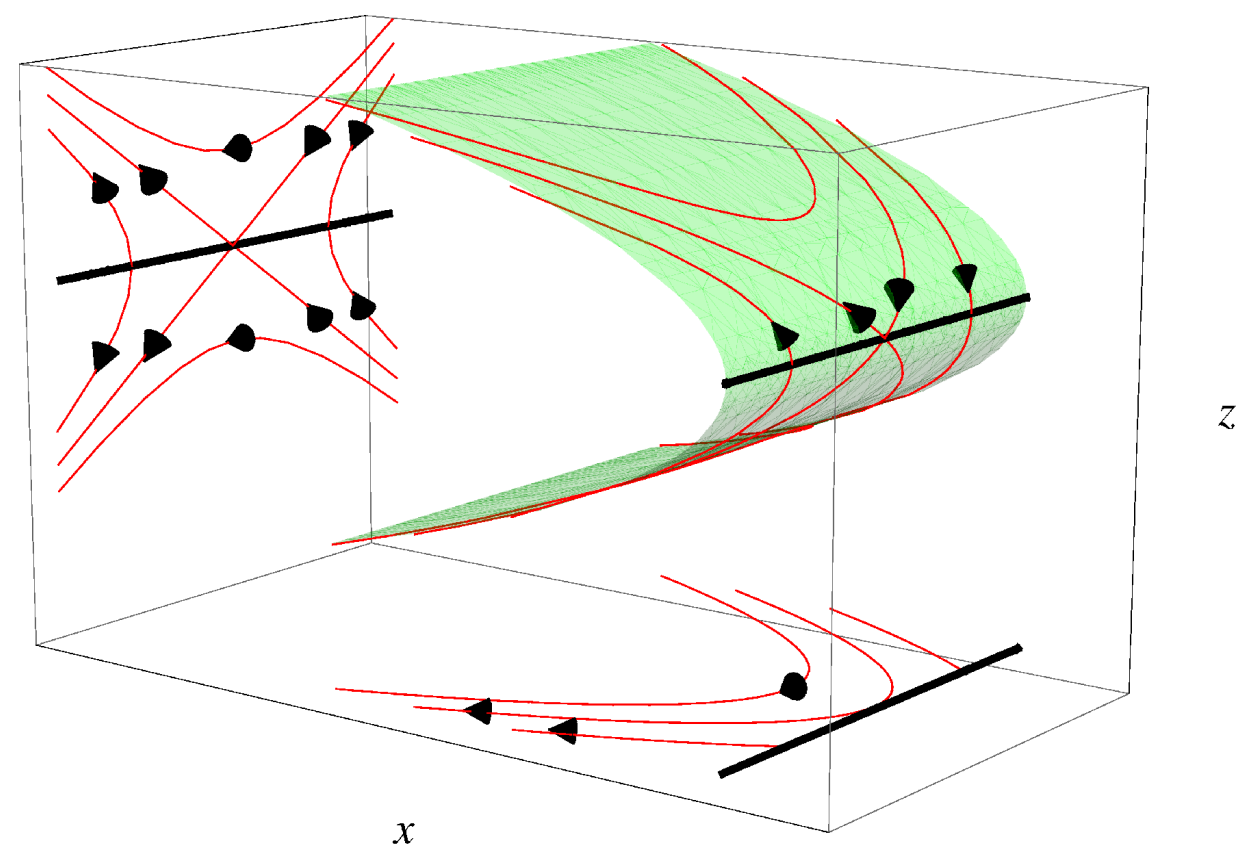

Figure 4: Folded saddle, corresponds to Figure 3, Case 9 in [14].

which $g_{z}>0$ are defined as unstable. (This definition is motivated by the corresponding theory of singularly perturbed equations.) The cusp surface $x=x(y, z)$ is given by the graph of $z^{3}+y z+x=0$, with $x$ as a function of the base variables $y$ and $z$. The graph is triple-valued for $y<0$ and single-valued for $y>0$. Moreover, in the triple-valued regime, two of the branches are stable while the middle one is unstable. The normal form obtained by changing the sign in the last equation of (18) corresponds to the middle branch in the triple-valued regime being stable and the lower and upper branches unstable. This normal form is not of interest for our purposes. The dynamics of (18) are depicted in Figure 5, on the cusp surface and in two projections. Note that (B) holds, (C) fails, and (D) holds.

\subsection{Singularities for (8)}

We now return to (8) and analyze the singularities it possesses. Solving the constraint $0=z^{3}+y z+x$ for $x$, we obtain $x=-\left(z^{3}+y z\right)$. Differentiating with respect to $t$ and substituting $-1-y$ for $\dot{x}$, we obtain

$$
-\left(3 z^{2}+y\right) \dot{z}-\dot{y} z=-1-y .
$$

Substituting for $\dot{y}$, we obtain

$$
\left(3 z^{2}+y\right) \dot{z}=(y+1)+2(y+z) z
$$

In combination with the $\dot{y}$ equation, we obtain the reduced system

$$
\begin{aligned}
\dot{y} & =-2(y+z) \\
\left(3 z^{2}+y\right) \dot{z} & =(y+1)+2(y+z) z .
\end{aligned}
$$

Finally, desingularization yields

$$
\begin{aligned}
& \dot{y}=-2(y+z)\left(3 z^{2}+y\right) \\
& \dot{z}=(y+1)+2(y+z) z .
\end{aligned}
$$




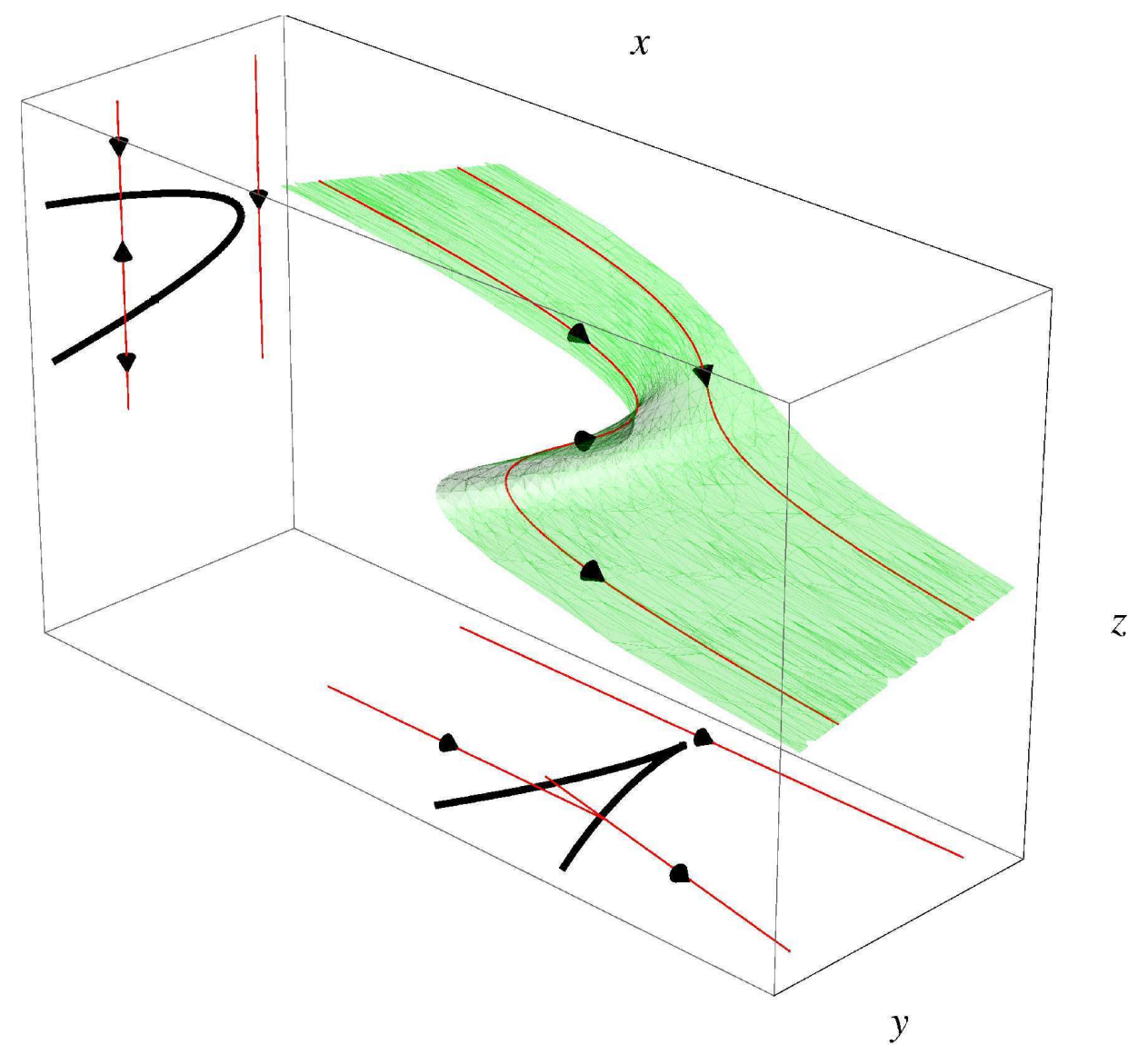

Figure 5: Dynamics of (18) with the plus sign, shown in the three-dimensional $x, y, z$ space as well as in two different projections. Corresponds to Figure 3, Case 11 in [14]. 


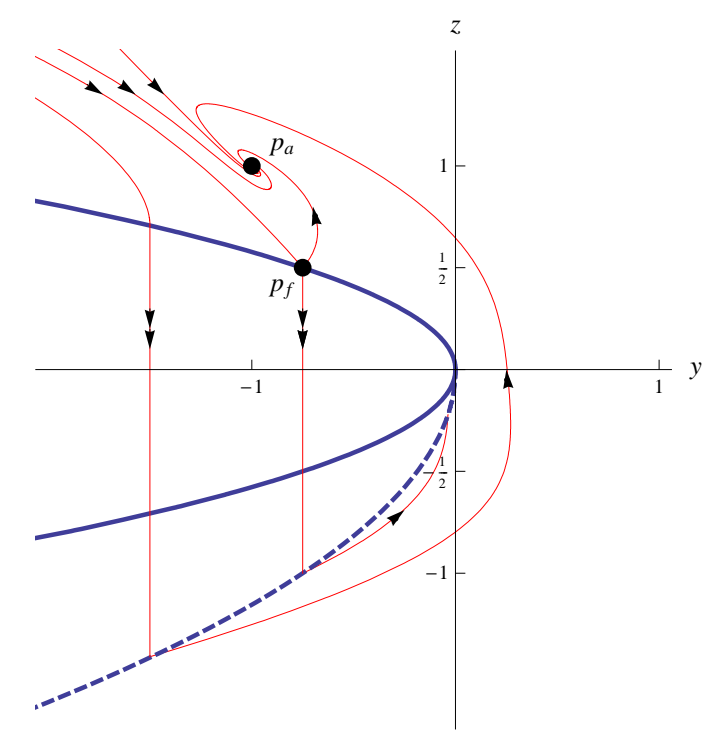

Figure 6: Phase portrait of the reduced equation (19)

The fold line is the parabola $y=-3 z^{2}$. Folded singularities are the equilibria of $(20)$ on the fold line, i.e. points satisfying the set of equations

$$
\begin{aligned}
& 0=y+3 z^{2} \\
& 0=(y+1)+2(y+z) z
\end{aligned}
$$

By eliminating $y$, we obtain $-6 z^{3}-z^{2}+1=0$. One readily verifies that $z=\frac{1}{2}$ is the only real root, with the corresponding value of $y=-\frac{3}{4}$. Hence, there is one folded singularity, given by $p_{f}=\left(-\frac{3}{4}, \frac{1}{2}\right)$. Linearizing (20) about $p_{f}$, we obtain the matrix

$$
A=\left(\begin{array}{cc}
\frac{1}{2} & \frac{3}{2} \\
2 & \frac{1}{2}
\end{array}\right) .
$$

Since $\operatorname{det} A<0$ the point $p_{f}$ is a folded saddle. Further, it is easy to check that the flow of (19) is away from the fold for $0<z<1 / 2$ and towards the fold for $z<0$ and for $z>1 / 2$. Figure 6 shows a computer generated phase portrait of (19).

\subsection{Singular global return for Zeeman's equation (8)}

The full fold line $F$ of equation (8) can be parametrized as follows:

$$
F=\left\{(x, y, z)=\left(2 \sigma^{3},-3 \sigma^{2}, \sigma\right): \sigma \in \mathbb{R}\right\} .
$$

The upper branch, denoted by $F_{+}$, corresponds to positive values of $z$, and the lower branch, $F_{-}$, corresponds to negative values of $z$. In this section, we look at the projection of $F_{+}$onto $F_{-}$.

The projection of $F_{+}$onto $S_{0}$ is obtained from (21) as the unique curve on the lower sheet, $F_{-}$, of $S_{0}$ with the property that the $x$ and $y$ coordinates remain unchanged. This curve has the following parametric representation:

$$
P F_{+}=\left\{(x, y, z)=\left(2 \sigma^{3},-3 \sigma^{2},-2 \sigma\right): \sigma>0\right\} .
$$

The $y$ and $z$ coordinates on $P F_{+}$satisfy the equation $y=-\frac{3}{4} z^{2}$.

The singular return is determined by the trajectories of (20) starting on $P F_{+}$. Figure 6 shows some of these trajectories, and the dashed curve represents $P F_{+}$. Some of the trajectories run into the fold line $F$ and can no longer stay on the constrained surface. Other trajectories pass to the right of the cusp, making 


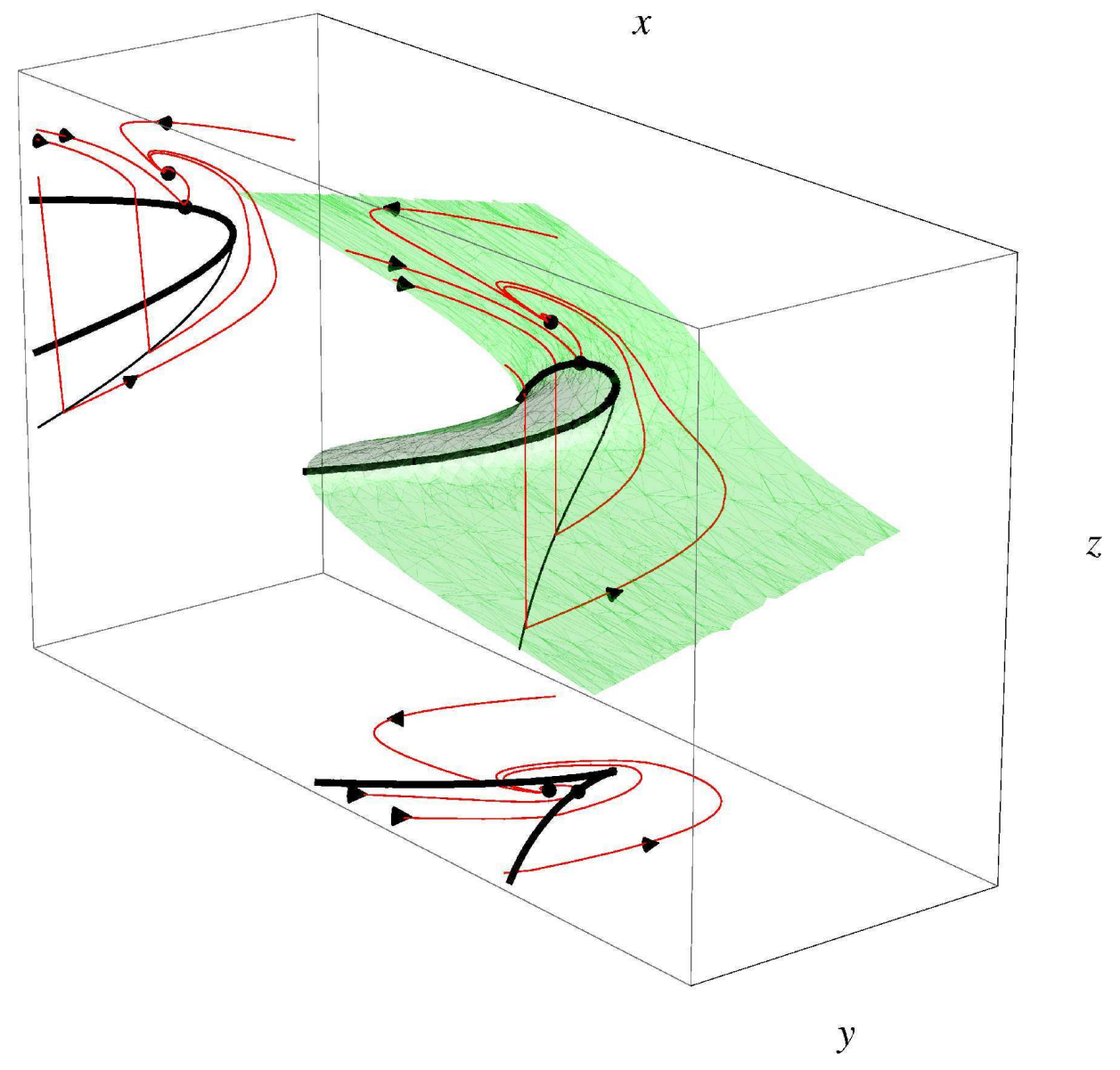

Figure 7: Phase portrait of the constrained equation (8) on the constraining manifold and in two projections. 


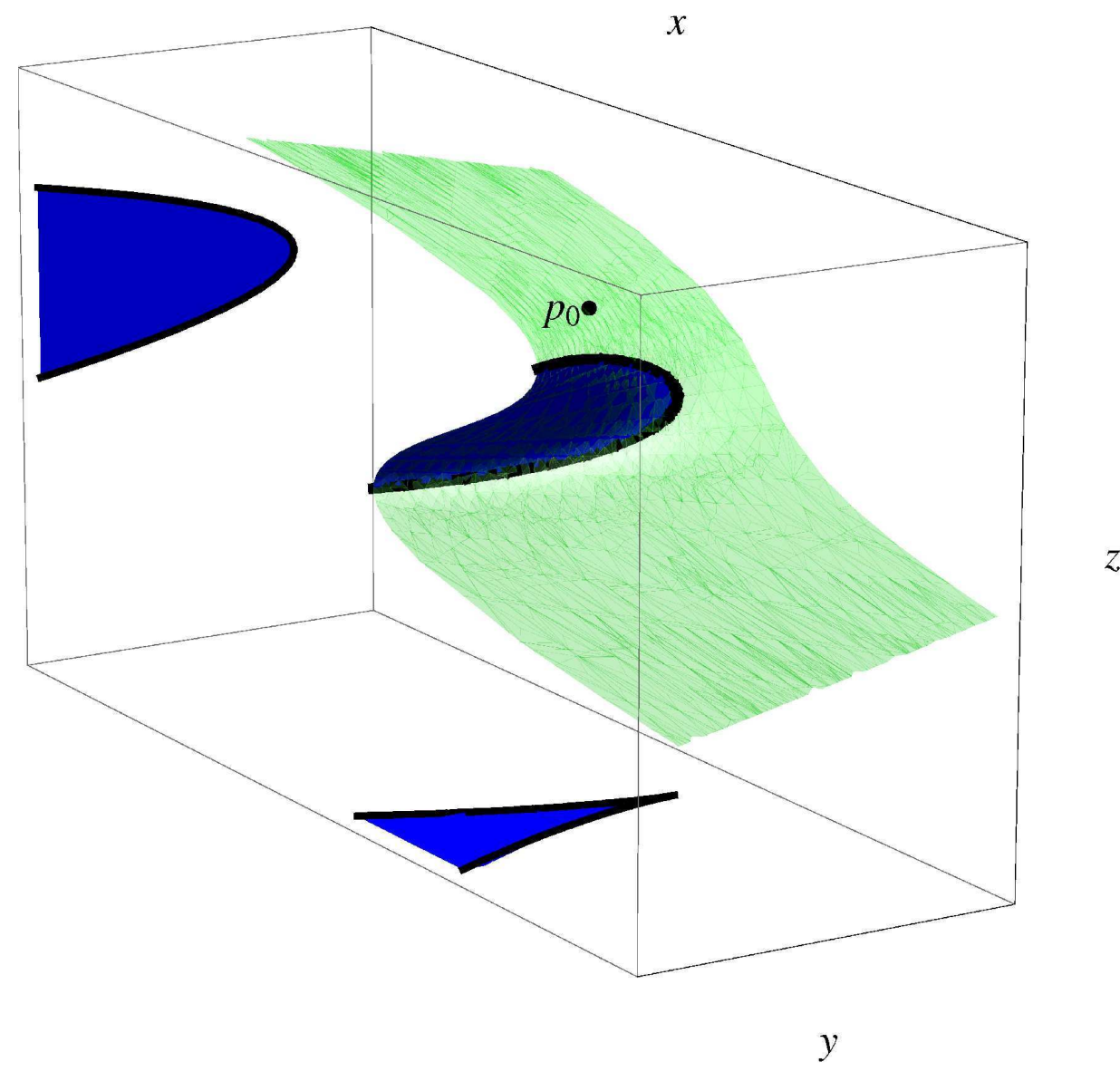

Figure 8: The manifold $S_{0}^{a}$.

a smooth return. Very important is the forward trajectory of the projection of $p_{f}$, which we denote by $q_{f}$. Note that $q_{f}=\left(-\frac{3}{4},-1\right)$. In the sequel we will characterize the dynamics of $(20)$. In particular we will show that the trajectory of $q_{f}$ passes through the lower branch of the fold line $F_{-}$(see Figure 6 ), and thereby does not correspond to a smooth return.

Let $S_{0}$ be the critical manifold for $(7)$ and let

$$
S_{0}^{a}=\left\{(x, y, z) \in S_{0}: \frac{\partial g}{\partial z}(x, y, z)<0\right\} .
$$

Further, let $S_{0-}^{a}$ (resp. $S_{0+}^{a}$ ) be $S_{0}^{a} \cap\{(x, y, z): z<0\}$ (resp. $S_{0}^{a} \cap\{(x, y, z): z \geq 0\}$ ). The manifold $S_{0}^{a}$ is shown in Figure 8.

Theorem 1 There exist sets $V_{01}, V_{02}$ and $V_{03}$ contained in $S_{0+}^{a}$ with the following properties:

(i) All trajectories of (19) starting in $V_{01}$ remain in $V_{01}$ and are attracted to the stable equilibrium $p_{a}$.

(ii) All trajectories of (19) starting in $V_{02}$ reach $F_{+}$, jump to $P F_{+}$, continue to $F_{-}$and jump to $V_{01}$.

(iii) All trajectories of (19) starting in $V_{03}$ reach $F_{+}$, jump to $P F_{+}$and continue to $V_{01}$.

Theorem 1 is a consequence of the following proposition:

Proposition 1 There exists a point $q_{*}=\left(y_{*}, z_{*}\right) \in P F^{+}$, with $z_{*}<-1$ whose forward trajectory under (20) passes through 0 . If $q \in\left\{(y, z) \in P F^{+}: z \leq-1\right\}$ and $q \neq q^{*}$ then one of the following cases holds: 


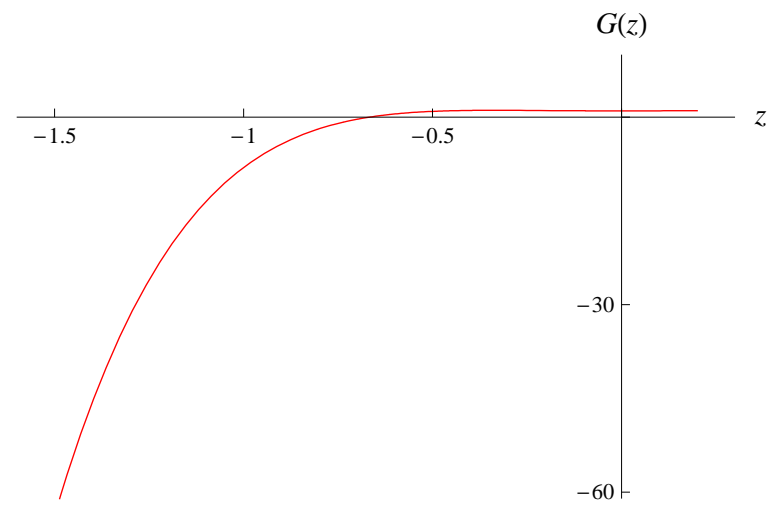

Figure 9: Graph of the inner product $G(z)$ as a function of $z$.

$z_{*}<z<-1$ The forward trajectory of $q$ under (20) passes through $F_{-}$.

$z<z_{*}$ The forward trajectory of $q$ under (20) passes to the right of the origin and enters the second quadrant through the positive $z$ axis.

In the proof of Proposition 1 we will rely on the following fact:

Lemma 2 The function

$$
G(z)=-\frac{27}{4} z^{4}\left(1-\frac{3}{4} z\right)-\frac{3}{2} z^{3}+\frac{5}{4} z^{2}+1
$$

is negative for $z<-1$

Proof The function $G$ can be rewritten as follows:

$$
G(z)=-\frac{27}{4} z^{4}\left(1-\frac{3}{4} z\right)-\frac{3}{2} z^{3}+\frac{5}{4} z^{2}+1 .
$$

First, observe that $G(-1)=-\frac{129}{16}<0$. Second, observe that

$$
G^{\prime}(z)=\frac{405}{16} z^{4}-\frac{z}{2}\left[54 z^{2}+9 z-5\right] .
$$

In this expression, the first term is positive for all $z<-1$. Also, the term in square brackets is positive for all $z<-1$, because both roots of this quadratic lie to the right of -1 . Hence, $G^{\prime}(z)>0$ for all $z<-1$, and the lemma is proven.

The graph of $G$ computed using MATHEMATICA is shown in Figure 9.

Proof of Proposition 1 Equation (20) restricted to $P F_{-}$, i.e., at a point $\left(z,-\frac{3}{4} z^{2}\right)$, is given by

$$
\begin{aligned}
& \dot{y}=-\frac{9}{2} z^{3}\left(1-\frac{3}{4} z\right) \\
& \dot{z}=-\frac{3}{2} z^{3}+\frac{5}{4} z^{2}+1 .
\end{aligned}
$$

A normal vector to $P F_{-}$at a point $\left(z,-\frac{3}{4} z^{2}\right)$ is $\left(\frac{3}{2} z, 1\right)$. Computing the inner product of $\left(\frac{3}{2} z, 1\right)$ with the vector field (23), we find the function $G(z)$ introduced in the statement of Lemma 2 . By Lemma $2 G(z)<0$ for $z<-1$. It follows that all the trajectories starting at points $q=(y, z) \in P F_{+}$must enter the region bounded by $\left\{(y, z) \in P F_{+}: z \leq-1\right\}$, the segment of the trajectory of $q_{f}$ from $q_{f}$ to the $y$ axis (see Figure $6)$ and the $y$ axis. Consider the trajectory starting at $\left\{(y, z) \in P F_{+}: z \leq-1\right\}$. Our goal is to prove that this trajectory reaches the $y$ axis. Note that $\dot{y}>0$ as long as $y+z<0$ so that $y$ initially keeps increasing, eventually passing the line $y+1=0$. Henceforth, $z$ also must be increasing until either the $y$ axis, or the line 
$y+z=0$ is reached. After the trajectory has crossed the line $y+z=0$ the $y$ coordinate must be decreasing but $y+z$ must stay positive as long as $z$ is negative. It follows that eventually the trajectory must reach the $y$ axis. The result follows by monotonicity of the flow.

\section{Singularly perturbed cusps - statement of the main theorem}

As mentioned, the main technical content of this article is the study of slow passage through the cusp for $0<\varepsilon \ll 1$ by means of geometric singular perturbation theory. Recall that the result establishing the equivalence of a system in general position to the normal form was proved in [14] for $\varepsilon=0$ only. Rather than considering the normal form, we consider a general system for the cusp singularity and study its dynamics. We consider systems in the fast formulation and add the equation $\varepsilon^{\prime}=0$ (this is standard in geometric singular perturbation theory, see, e.g., [8]),

$$
\begin{aligned}
x^{\prime} & =\varepsilon f_{1}(x, y, z, \varepsilon) \\
y^{\prime} & =\varepsilon f_{2}(x, y, z, \varepsilon) \\
z^{\prime} & =g(x, y, z, \varepsilon), \\
\varepsilon^{\prime} & =0,
\end{aligned}
$$

satisfying the defining conditions (10), the condition (B), the condition $g_{z z}\left(x_{0}, y_{0}, z_{0}\right)=0$, which is a violation of $(\mathrm{C})$, and condition (D). We assume without loss of generality that $\left(x_{0}, y_{0}, z_{0}\right)=(0,0,0)$. We restate the defining and non-degeneracy conditions (for convenience of the reader)

$$
\begin{aligned}
& g(0,0,0,0)=0 \\
& g_{z}(0,0,0,0)=0 \\
& g_{z z}(0,0,0,0)=0
\end{aligned}
$$

and the nondegeneracy conditions

$$
\begin{aligned}
& g_{z z z}(0,0,0,0) \neq 0 \\
& \left(g_{x}(0,0,0,0), g_{y}(0,0,0,0)\right) \cdot f(0,0,0,0) \neq 0 .
\end{aligned}
$$

We prove the following result:

Proposition 2 Assume (25) and (26). Then, in a sufficiently large neighborhood of the origin, (24) can be transformed to new variables in which $f_{1}(0,0,0,0) \neq 0, f_{2}(0,0,0,0)=0$ and

$$
g(x, y, z, \varepsilon)=-x+a_{1}(x, y, \varepsilon) z+a_{2}(x, y, \varepsilon) z^{3}+O\left(z^{4}\right) .
$$

Proof First, we translate the $z$ variable to eliminate the $z^{2}$ term in $g$. Next, we note that (26) implies that $\left(g_{x}(0,0,0,0), g_{y}(0,0,0,0)\right) \neq(0,0)$. To fix ideas we assume $g_{x}(0,0,0,0) \neq 0$. We replace $x$ by a new variable,

$$
w=-g(x, y, 0, \varepsilon) .
$$

After these transformations, (24) becomes

$$
\begin{aligned}
w^{\prime} & =-\varepsilon\left(g_{x}(w, y, 0, \varepsilon), g_{y}(w, y, 0, \varepsilon)\right) \cdot f(w, y, z, \varepsilon) \\
y^{\prime} & =\varepsilon f_{2}(w, y, z, \varepsilon) \\
z^{\prime} & =g(w, y, z, \varepsilon) \\
\varepsilon^{\prime} & =0
\end{aligned}
$$

with the transformed $g$ now having the form (27). We conclude the proof by letting

$$
y \rightarrow y+\frac{f_{2}(0,0,0,0)}{\left(g_{x}(0,0,0,0), g_{y}(0,0,0,0)\right) \cdot f(0,0,0,0)} w .
$$


The transformed system (28) now has the desired properties.

An additional non-degeneracy condition is needed concerning (24) for our later analysis, namely we assume

$$
a_{1, y}(0,0,0) \neq 0
$$

Further, we assume

$$
a_{2}(0,0,0)<0, \quad\left(g_{x}(0,0,0,0), g_{y}(0,0,0,0)\right) \cdot f(0,0,0,0)<0 .
$$

These assumptions define one of the generic cases, which is the one occurring for the nerve impulse equation, leading to Figure 3 Case 11 in [14]. Also, without loss of generality, we assume $a_{1, y}(0,0,0)<0$ (if the opposite holds we let $y \rightarrow-y)$. By scaling the variables, we make $f_{1}(0,0,0)=1,, a_{1, y}(0,0,0)=-1$ and $g_{z z z}(0,0,0,0)=-1$.

Note that, after all the transformations and assumptions, (24) can be written in the following form:

$$
\begin{aligned}
& x^{\prime}=\varepsilon(1+O(x, y, z, \varepsilon)) \\
& y^{\prime}=\varepsilon O(x, y, z, \varepsilon) \\
& z^{\prime}=-\left(z^{3}+y z+x\right)+O\left(\varepsilon, x z, y z^{3}, z^{4}\right) .
\end{aligned}
$$

This is the form of the system with which we work throughout the rest of this section, and we note that, to leading order, (31) is the same as the fast version of (18).

We are interested in describing the transition between the sections

$$
\Sigma^{\text {in }}=\left\{(x, y, z): x=-x_{0}, z>0\right\},
$$

and

$$
\Sigma^{\text {out }}=\left\{(x, y, z): x=x_{0}\right\}
$$

where $x_{0}>0$ is a small constant. Since we want to study passage near the cusp point, we consider only the initial conditions which, before the arrival to $\Sigma^{\text {in }}$, were attracted to the slow manifold $S_{a, \varepsilon}$. This justifies the condition $z>0$ in the definition of $\Sigma^{\text {in }}$. The sections of interest are shown in Figure 10 (see also Figure 5). Note that by Theorem 5 there exist slow manifolds $S_{\varepsilon}^{-}$and $S_{\varepsilon}^{+}$obtained as small perturbations of the sets

$$
\left\{(x, y, z) \in S_{0}: x \leq-x_{0}<0\right\}
$$

and

$$
\left\{(x, y, z) \in S_{0}: x \geq x_{0}>0\right\}
$$

respectively, with $x_{0}$ an arbitrary constant.

The following theorem is main result of this article:

\section{Theorem 2 (Cusp)}

For system (24) with assumptions (25), (26), (29), and (30), there exists an $\varepsilon_{0}>0$ sufficiently small such that for all $0<\varepsilon \leq \varepsilon_{0}$, the following statements hold:

(i) The transition map $\Pi: \Sigma^{i n} \rightarrow \Sigma^{\text {out }}$ induced by the flow of (31) is a diffeomorphism mapping a rectangular neighborhood of $S_{0}$ into $\Sigma^{\text {out }}$.

(ii) The choice of $S_{\varepsilon}^{+}$can be made in such a way that $\Pi\left(\Sigma^{i n} \cap S_{\varepsilon}^{-}\right) \subset S_{\varepsilon}^{+}$.

(iii) The map $\Pi$ is exponentially contracting in the $z$ direction and the derivative in the $y$ direction is bounded above and below. More precisely,

$$
\left|\frac{\partial \Pi}{\partial z}\right|=O\left(e^{-c / \varepsilon}\right)
$$

for some positive constant $c$ and there exists a constant $K>0$ such that

$$
\frac{1}{K} \leq\left|\frac{\partial \Pi}{\partial y}\right| \leq K
$$

The proof of Theorem 2 will be given in the next section. 


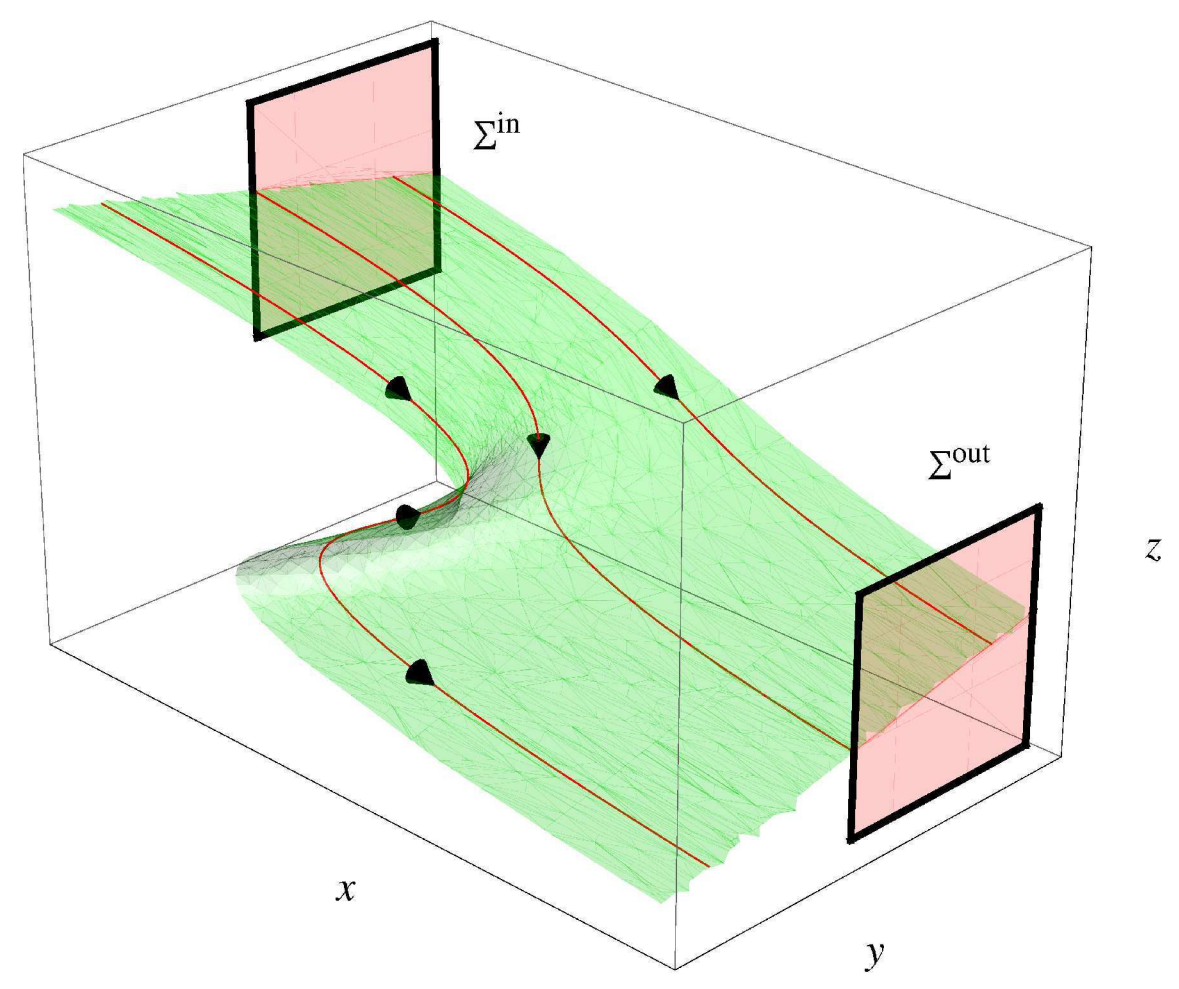

Figure 10: The extremal sections $\Sigma^{\text {in }}$ and $\Sigma^{\text {out }}$.

\section{Analysis of a singularly perturbed cusp by means of geometric desingularization}

Note $(x, y, z, \varepsilon)=(0,0,0,0)$ is a degenerate equilibrium with zero as a triple eigenvalue of (31). In the neighborhood of this equilibrium, the distinction between fast and slow variables is lost, and the analysis is further complicated by the fact that the two fold lines of the cusp surface, which themselves are already not normally hyperbolic, meet at the cusp point. Nevertheless, the system dynamics may be analyzed using the method of geometric desingularization, also known as the blowup method $[2,3,4,5,10,11]$. Here, the origin is blown up into a hyper-sphere, and the induced equilibria are either hyperbolic or semi-hyperbolic. As a result, standard invariant manifold theory may be used to analyze the dynamics of the transformed vector field.

Let $\Phi: \mathbb{R}^{5} \rightarrow \mathbb{R}^{4}$ be defined by

$$
x=r^{3} \bar{x}, \quad y=r^{2} \bar{y}, \quad z=r \bar{z}, \quad \varepsilon=r^{5} \bar{\varepsilon} .
$$

Let $S^{3} \equiv\left\{(\bar{x}, \bar{y}, \bar{z}, \bar{\varepsilon}) \mid \bar{x}^{2}+\bar{y}^{2}+\bar{z}^{2}+\bar{\varepsilon}^{2}=1\right\}$, and let $\rho_{0}$ be determined by the relation $\varepsilon_{0}=\rho_{0}^{5}$. Then, define $B=S^{3} \times\left[0, \rho_{0}\right]$. The blow-up transformation (32) is the restriction of $\Phi$ to $B$. Let $\mathbf{X}$ denote the original vector field (24) and let $\overline{\mathbf{X}}$ be defined by $\Phi_{*} \overline{\mathbf{X}}=\mathbf{X}$, where $\Phi_{*}$ is induced by $\Phi$. Vector field $\bar{X}$ is (smoothly) conjugate to $X$ on $S^{3} \times\left(0, \rho_{0}\right]$ and is 0 on $S^{3} \times\{0\}$. The essence of the blow-up method is to rescale $\bar{X}$ in such a way that the limit of the rescaled version of $\bar{X}$ on $S^{3} \times\{0\}$ is non-trivial and contains vital information about the flow of $X$. In fact, in order to highlight different features of the flow, one uses different scalings in different parts of $B$. For this reason the blow up of $X$ is sometimes referred to as a local vector field.

Our principal objective is to study the blow-p of $X$ on $B$. As is common in differential geometry when working with spheres, it is convenient to employ charts, rather than spherical coordinates. Hence, we 

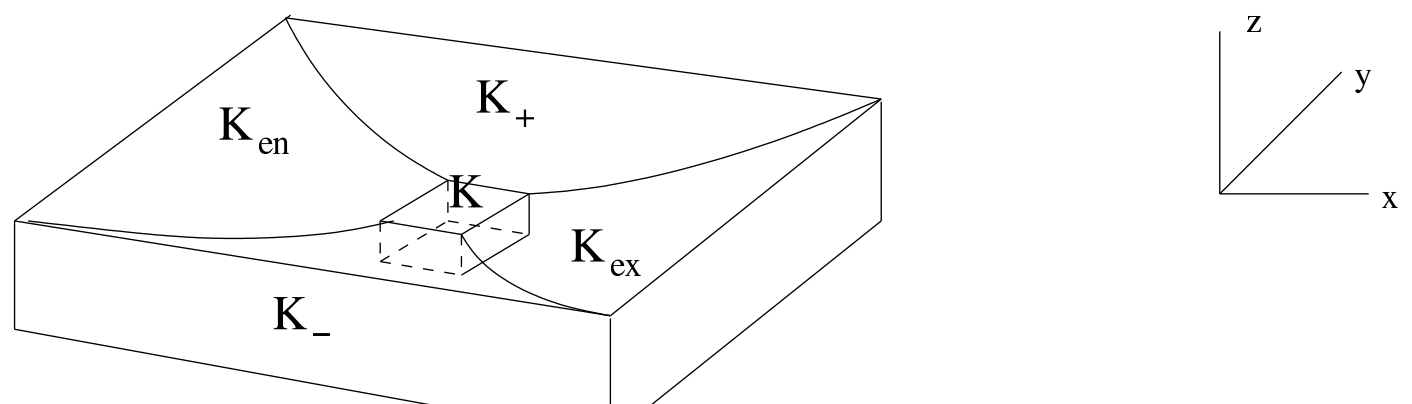

Figure 11: The domains of the charts $K_{\mathrm{en}}, K, K_{-}, K_{+}$, and $K_{\mathrm{ex}}$ in the original variables.

introduce the following hyperplanes in $\mathbb{R}^{5}$, which will serve as charts for different parts of $B$ :

$$
\begin{aligned}
& K_{\mathrm{en}}:\{\bar{x}=-1\} \\
& K:\{\bar{\varepsilon}=1\} \\
& K_{-}:\{\bar{y}=-1\} \\
& K_{+}:\{\bar{y}=1\} ; \\
& K_{\mathrm{ex}}:\{\bar{x}=1\}
\end{aligned}
$$

In these charts, we track trajectories as they enter a neighborhood of the origin, pass through it, and exit it. Therefore, we refer to them as the entry -, rescaling -, and exit charts. In Figure 11, we show the domain of the charts $K_{\mathrm{en}}, K, K_{-}, K_{+}$, and $K_{\mathrm{ex}}$ in the original variables. The union of these domains is a neighborhood of the cusp point which is uniform (independent of $\varepsilon$ ) in the $x$ and $y$ directions but in the $z$ direction shrinks to $O\left(\varepsilon^{1 / 3}\right)$ near the cusp. All trajectories which are attracted to the left branch of the slow manifold for $\varepsilon$ and $y$ sufficiently small (with the bound on $y$ independent of $\varepsilon$ ) must pass through this neighborhood, entering through the domain of the entry chart and exiting through the domain of the exit chart.

We will define a number of sections of the flow in the charts; the first being the equivalent of $\Sigma^{\text {in }}$ in $K_{\text {en }}$. All the subsequently defined sections are determined by the flow in the charts. First, we define an 'out' section in $K_{\mathrm{en}}$, which gives an 'in' section in $K$. Next, we define an 'out' section in $K$, etc...

We begin in Section 4.1 where we study the passage of trajectories through chart $K_{\mathrm{en}}$, which in terms of the original vector field corresponds to entering a small fixed neighborhood of the cusp point and continuing until $x=O\left(\varepsilon^{1 / 3}\right), y=O\left(\varepsilon^{1 / 2}\right)$ and $z=O(\varepsilon)$. Then, in Sections 4.2 and 4.3, we study the dynamics in chart $K$, which corresponds to the dynamics in a neighborhood of size $O\left(\varepsilon^{1 / 3}\right) \times O\left(\varepsilon^{1 / 2}\right) \times O(\varepsilon)$ for $(31)$; the distinguished limit of the dynamics near the cusp point is identified herein. Finally, in Section 4.4, we analyze the system dynamics in the exit chart $K_{\text {ex }}$ which corresponds to moving from $O\left(\varepsilon^{1 / 3}\right) \times O\left(\varepsilon^{1 / 2}\right) \times O(\varepsilon)$ to a uniformly bounded distance away from the origin (for the variables $(x, y, z)$ ), and in Section 4.5 we prove Theorem 2.

Remark. The scalings in (32) were found by requiring that all of the terms in the transformed vector field involve the same power of $r$.

\subsection{Dynamics in the entry chart $K_{\mathrm{en}}$}

In the section, we analyze the dynamics of (31) in the entry chart $K_{\text {en }}$ and use the subscript 1 on the variables (dropping the overbars) in order to indicate that the analysis is for this chart. This notational convention extends to the variables in the other charts, as is customary.

Recall the definition of chart $K_{\text {en }}$ given by (33). It follows that the rescalings (32) become

$$
x=-r_{1}^{3}, \quad y=r_{1}^{2} y_{1}, \quad z=r_{1} z_{1}, \quad \varepsilon=r_{1}^{5} \varepsilon_{1} .
$$

We transform (31) to variables (34) and apply a time rescaling, cancelling a factor of $r_{1}^{2}$. The resulting 


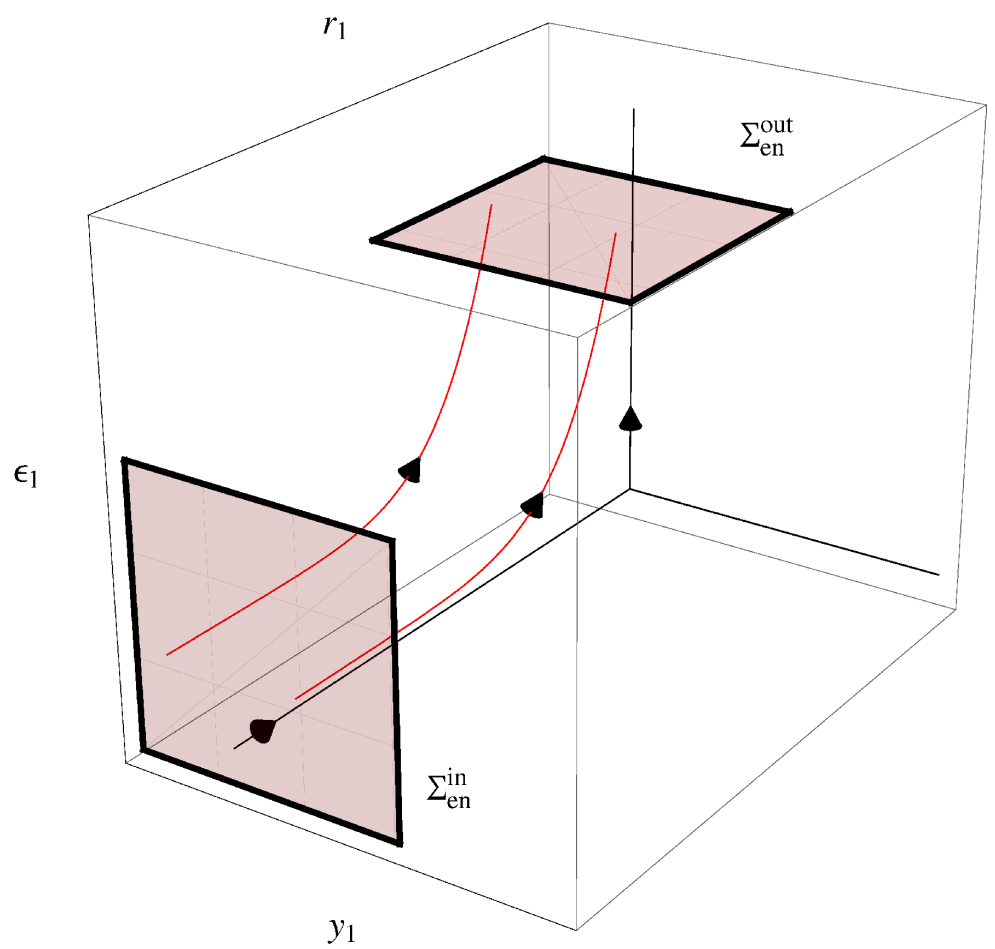

Figure 12: The sections $\Sigma_{\mathrm{en}}^{\text {in }}$ and $\Sigma_{\mathrm{en}}^{\text {out }}$, with $z_{1}$ suppressed.

system is

$$
\begin{aligned}
& \dot{r}_{1}=-\frac{1}{3} \varepsilon_{1} r_{1}\left(1+O\left(r_{1}\right)\right) \\
& \dot{y}_{1}=\frac{2}{3} \varepsilon_{1} y_{1}+O\left(r_{1} \varepsilon_{1}\right) \\
& \dot{z}_{1}=-\left(z_{1}^{3}+y_{1} z_{1}-1-\frac{1}{3} \varepsilon_{1} z_{1}\right)+O\left(r_{1}\right) \\
& \dot{\varepsilon}_{1}=\frac{5}{3} \varepsilon_{1}^{2}\left(1+O\left(r_{1}\right)\right) .
\end{aligned}
$$

We note that, although the time variable has been rescaled, the overdot has been recycled for convenience of notation.

We now define the following sections of the flow:

$$
\Sigma_{\mathrm{en}}^{\mathrm{in}} \equiv\left\{\left(r_{1}, y_{1}, z_{1}, \varepsilon_{1}\right) \mid r_{1}=\rho, z_{1}>0\right\}
$$

and

$$
\Sigma_{\mathrm{en}}^{\mathrm{out}} \equiv\left\{\left(r_{1}, y_{1}, z_{1}, \varepsilon_{1}\right) \mid \varepsilon_{1}=\delta\right\}
$$

where $\rho=\sqrt[3]{x_{0}}$ and $\delta$ is a sufficiently small constant. We note that $\Sigma_{\text {en }}^{\text {in }}$ is equivalent to $\Sigma^{\text {in }}$. In Figure 12 , we show the sections $\Sigma_{\text {en }}^{\text {in }}$ and $\Sigma_{\text {en }}^{\text {out }}$, suppressing the variable $z_{1}$.

The goal of the analysis in this section is to describe the transition from $\Sigma_{1}^{\text {in }}$ to $\Sigma_{\text {en }}^{\text {out }}$. System (35) has several properties. The hyperplanes $\left\{r_{1}=0\right\}$ and $\left\{\varepsilon_{1}=0\right\}$ are invariant. Consequently, the $2 \mathrm{D}$ space $\left\{r_{1}=\varepsilon_{1}=0\right\}$ is also invariant. The dynamics in this space organizes the dynamics of the whole system. Restricted to the space $\left\{r_{1}=\varepsilon_{1}=0\right\}$, system (35) becomes the following planar system with horizontal flow:

$$
\begin{aligned}
& \dot{y}_{1}=0 \\
& \dot{z}_{1}=-\left(z_{1}^{3}+y_{1} z_{1}-1\right) .
\end{aligned}
$$




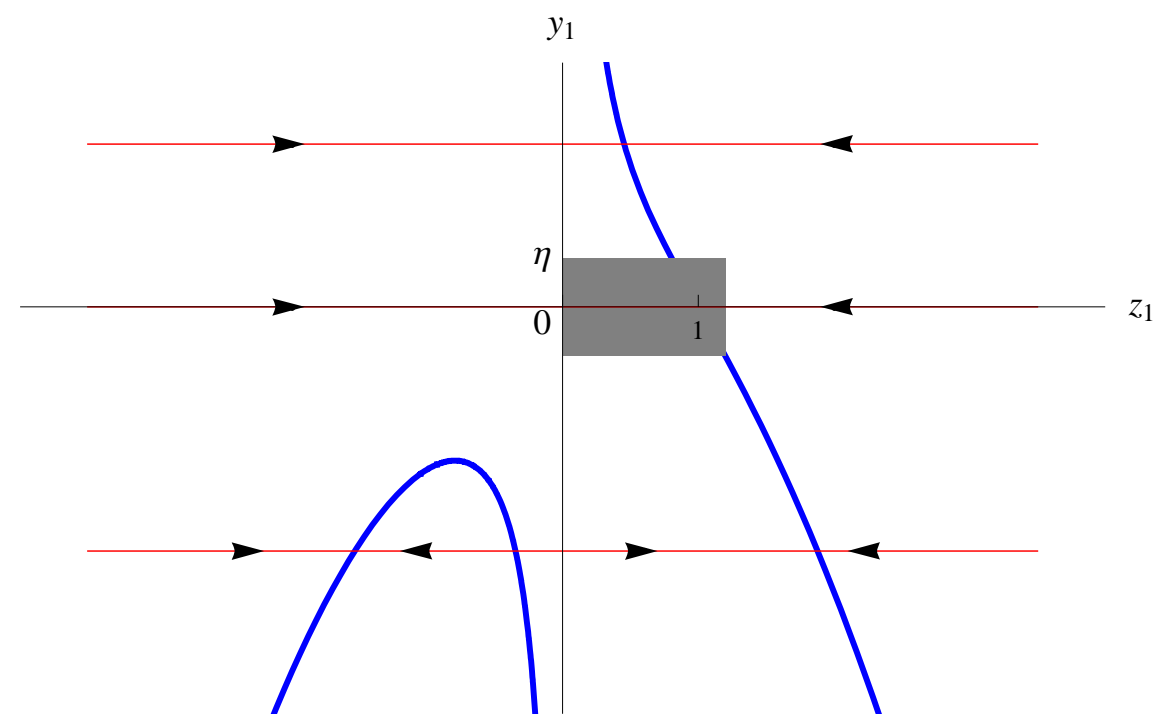

Figure 13: Dynamics in chart $K_{\mathrm{en}}$.

Now, we can understand the dynamics of (35) based on the phase portrait of the horizontal flow of (38). We note that there are two curves of equilibria (shown in blue) determined by the equation $z_{1}^{3}+y_{1} z_{1}-1=0$. Further, the flow on the invariant, horizontal lines is as shown in Figure 13. The dynamics of (35) depends on the sign of $y_{1}$, see the second equation of (35) and Figure 13. As $\varepsilon_{1}$ steadily increases along trajectories, each trajectory starting in $\Sigma_{\mathrm{en}}^{\mathrm{in}}$ eventually arrives in $\Sigma_{\mathrm{en}}^{\text {out }}$. A calculation shows that if $y_{0}$ is the value of $y$ in $\Sigma_{\mathrm{en}}^{\text {in }}$ then $y_{1}=(\delta / \varepsilon)^{2 / 5} y_{0}$ in $\Sigma_{\mathrm{en}}^{\text {out }}$, since $d y_{1} / d \varepsilon_{1}=(2 / 5)\left(y_{1} / \varepsilon_{1}\right)$ to leading order, and since $y_{0}=\rho^{2} y_{1}$ and $\varepsilon=\rho^{5} \varepsilon_{1}$ on $\Sigma_{\mathrm{en}}^{\mathrm{in}}$. Plainly, if $y_{0}$ does not satisfy the estimate $y_{0}=O\left(\varepsilon^{2 / 5}\right)$ then the value of $y_{1}$ in $\Sigma_{\mathrm{en}}^{\text {out }}$ blows up. In this case, we use different outgoing sections, depending on the sign of $y$,

$$
\Sigma_{ \pm}^{\text {out }} \equiv\left\{\left(r_{1}, y_{1}, z_{1}, \varepsilon_{1}\right) \mid y_{1}= \pm \eta\right\}
$$

where $\eta>0$ is an arbitrary positive number.

\subsection{Dynamics in the rescaling chart $K$}

In this section, we examine the dynamics in the rescaling chart $K$ and use the subscript 2 on the variables. The rescalings (32) become

$$
x=r_{2}^{3} x_{2}, \quad y=r_{2}^{2} y_{2}, \quad z=r_{2} z_{2}, \quad \varepsilon=r_{2}^{5},
$$

We transform (31) using the variables (40) and apply a time rescaling (by a factor of $r_{2}^{2}$ ). The resulting system is

$$
\begin{aligned}
& \dot{r}_{2}=0 \\
& \dot{x}_{2}=1+O\left(r_{2}\right) \\
& \dot{y}_{2}=O\left(r_{2}^{2}\right) \\
& \dot{z}_{2}=-\left(z_{2}^{3}+y_{2} z_{2}+x_{2}\right)+O\left(r_{2}\right)
\end{aligned}
$$

where the overdot denotes the derivative with respect to the rescaled time.

We define the following sections:

$$
\Sigma_{2}^{\text {in }} \equiv\left\{\left(x_{2}, z_{2}, y_{2}\right): x_{2}=-\tilde{k}, z_{2}>0\right\}
$$

and

$$
\Sigma_{2}^{\text {out }} \equiv\left\{\left(x_{2}, z_{2}, y_{2}\right): x_{2}=\tilde{k}, z_{2}<0\right\}
$$


where $\tilde{k}$ is a sufficiently large number. The goal of this section is to describe the transition from $\Sigma_{2}^{\text {in }}$ to $\Sigma_{2}^{\text {out }}$.

In system (41), the variable $r_{2}$ is constant along the orbits and $y_{2}$ varies very slowly. Consequently, one can understand the dynamics by drawing the phase portrait in the $\left(x_{2}, z_{2}\right)$ plane, with $r_{2}$ and $y_{2}$ treated as parameters, $r_{2} \approx 0$. For each fixed, real value of $y_{2}$, the $z_{2}$ nullcline is given in the $\left(x_{2}, z_{2}\right)$ plane by the cubic curve

$$
x_{2}=-y_{2} z_{2}-z_{2}^{3} .
$$

For any $y_{2}<0$, the cubic nullcline intercepts the $z_{2}$-axis in three distinct points, whereas it does so exactly once for any $y_{2} \geq 0$, with a vertical tangency in the transition case of $y_{2}=0$. In fact, the union of these cubic nullclines (44) over all $y_{2} \in \mathbb{R}$ is precisely the leading order formula for the cusp surface.

In this chart, $x_{2}$ and $z_{2}$ evolve on the same time scale. This reflects the fact that $z_{2}$ is no longer fast near the cusp point, and that the distinguished limit is the one in which $x_{2}$ and $z_{2}$ are equally slow. Trajectories cross the cusp surface with a horizontal tangency (parallel to the $x_{2}$-axis) as they move from left to right. Furthermore, the $z_{2}$ variable increases to the left of the associated cubic, while it decreases to the right of it. As a result, for the folded portion of the cusp surface corresponding to $y_{2}<0$, orbits pierce through both the upper and lower branches from below to above, locally. The dynamics in the $\left(z_{2}, x_{2}\right)$ plane are illustrated for $y_{2}>0$ and $y_{2}<0$ in Figure 14. We conclude that all trajectories starting in $\Sigma_{2}^{\text {in }}$ arrive in $\Sigma_{2}^{\text {out }}$ (flowbox theorem).

Finally, we discuss the relation between the sections $\Sigma_{\mathrm{en}}^{\text {out }}$ and $\Sigma_{2}^{\mathrm{in}}$. To describe the transition between these two sections, we need the coordinate change $\kappa_{12}$ from $K_{\text {en }}$ to $K$ and its inverse, $\kappa_{21}=\kappa_{12}^{-1}$. These transformations are given by

$$
\begin{aligned}
& \kappa_{12}: x_{2}=-\varepsilon_{1}^{-3 / 5}, \quad y_{2}=y_{1} \varepsilon_{1}^{-2 / 5}, \quad z_{2}=z_{1} \varepsilon_{1}^{-1 / 5}, \quad r_{2}=r_{1} \varepsilon_{1}^{1 / 5} ; \\
& \kappa_{21}: y_{1}=y_{2} x_{2}^{-2 / 3}, \quad z_{1}=-z_{2} x_{2}^{-1 / 3}, \quad \varepsilon_{1}=-x_{2}^{-5 / 3}, \quad r_{1}=-r_{2} x_{2}^{1 / 3} .
\end{aligned}
$$

It now follows that if we define

$$
\Sigma_{2}^{\text {in }}=\kappa_{12}\left(\Sigma_{\mathrm{en}}^{\mathrm{out}}\right)
$$

then $\tilde{k}=\delta^{-3 / 5}$.

\subsection{Dynamics in the rescaling charts $K_{-}$and $K_{+}$}

In this section, we examine the dynamics in the rescaling chart $K_{-}$. As the dynamics in $K_{+}$is much simpler we leave the analysis to the reader (it is possible to use a similar approach as we use for $K_{-}$in this section). We use the subscript 2 on the variables (dropping the overbars), as in Section 4.2. Here, the rescalings (32) become

$$
x=r_{2}^{3} x_{2}, \quad y=-r_{2}^{2}, \quad z=r_{2} z_{2}, \quad \varepsilon=r_{2}^{5} \varepsilon_{2} .
$$

We transform (31) using the variables (45) and apply a time rescaling (by a factor of $r_{2}^{2}$ ). The resulting system is

$$
\begin{aligned}
\dot{x}_{2} & =\varepsilon_{2}\left(1+O\left(r_{2}\right)\right)+\frac{3}{2} r_{2} x_{2} \varepsilon_{2} O\left(r_{2}\right) \\
\dot{r}_{2} & =-\frac{1}{2} r_{2}^{2} \varepsilon_{2} O\left(r_{2}\right) \\
\dot{z}_{2} & =-\left(z_{2}^{3}-z_{2}+x_{2}\right)+\frac{1}{2} r_{2} z_{2} \varepsilon_{2} O\left(r_{2}\right) \\
\dot{\varepsilon}_{2} & =\frac{5}{2} r_{2} \varepsilon_{2}^{2} O\left(r_{2}\right),
\end{aligned}
$$

where the overdot denotes the derivative with respect to the rescaled time. Note that $r_{2}=0$ defines an invariant space on which the dynamics are given by

$$
\begin{aligned}
& \dot{x}_{2}=\varepsilon_{2} \\
& \dot{z}_{2}=-\left(z_{2}^{3}-z_{2}+x_{2}\right) \\
& \dot{\varepsilon}_{2}=0 .
\end{aligned}
$$



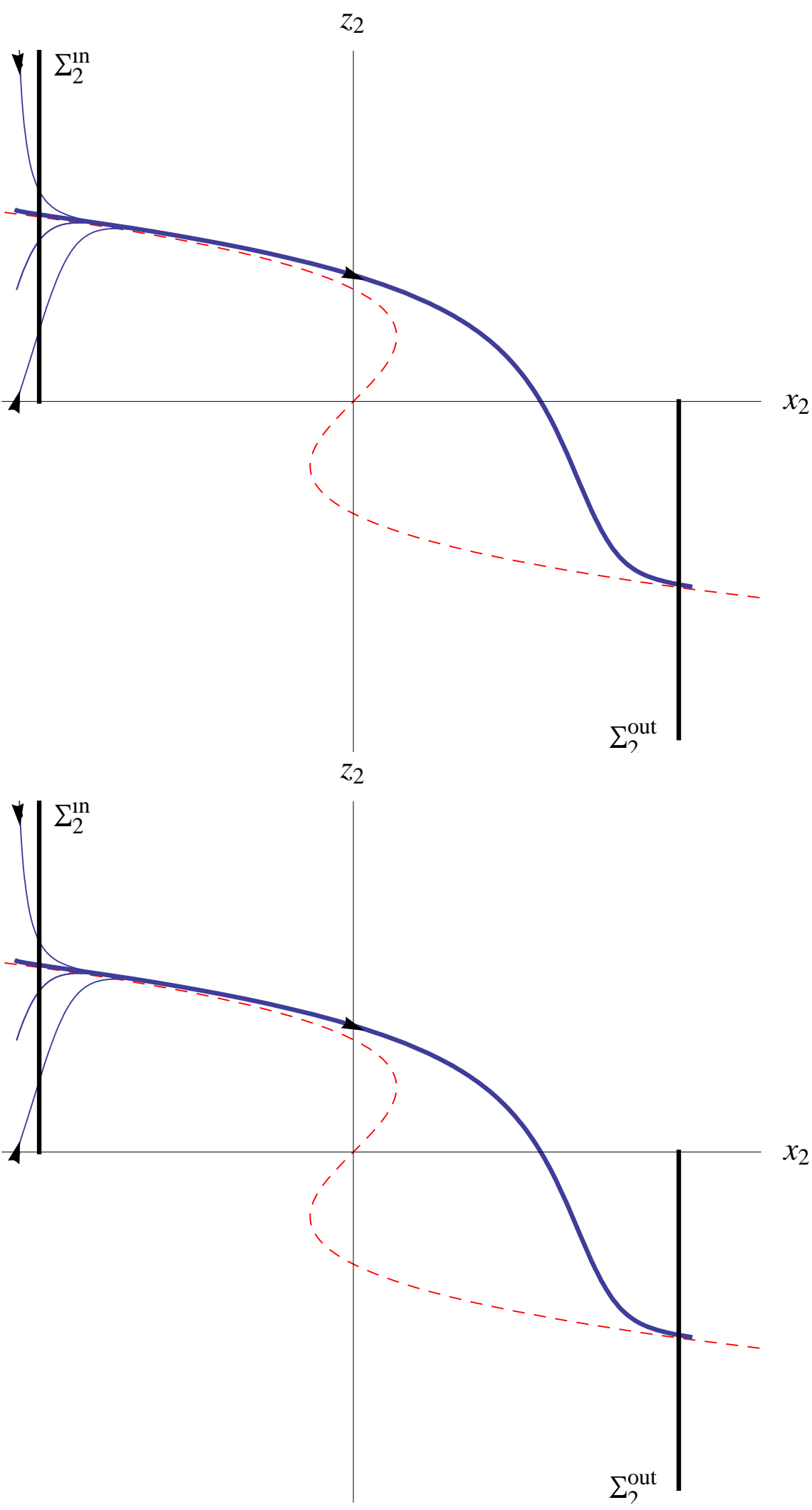

Figure 14: Dynamics in chart $K$. In the top frame, $y_{2}>0$; and in the bottom frame $y_{2}<0$. The dashed curves denote the $z_{2}$-nullcline. 


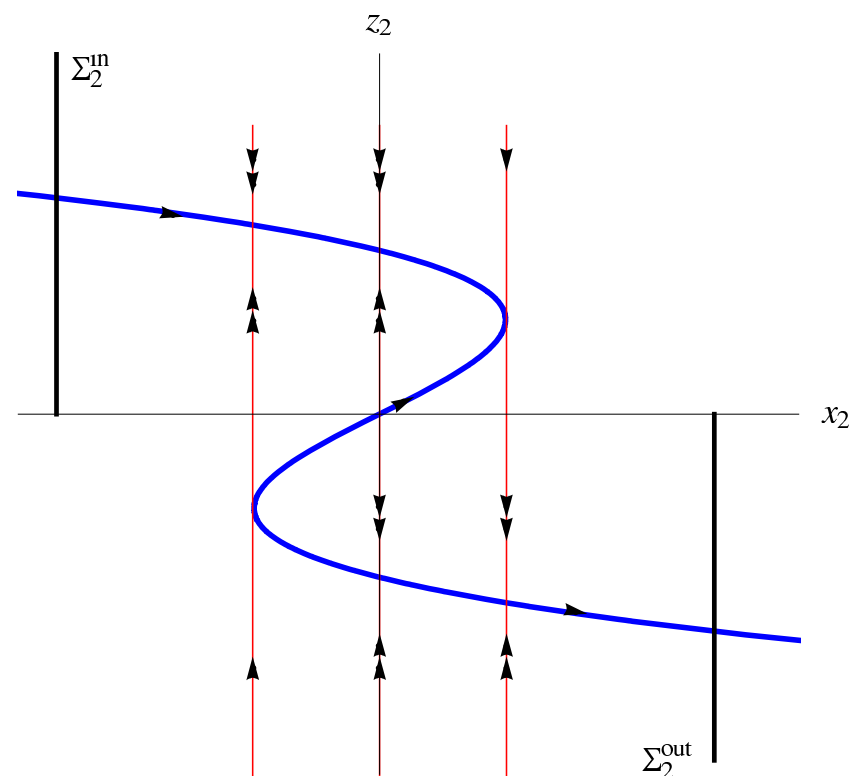

Figure 15: Slow/fast dynamics of (47); two folds subordinate to a cusp.

We assume that $|y| \leq \rho^{2}$, which implies $\eta^{1 / 2}(\varepsilon / \delta)^{1 / 5}<r_{2}<\rho$. As a consequence, we derive a bound for $\varepsilon_{2}$, namely $0<\varepsilon_{2}<\delta / \eta^{5 / 2}$. Since we can pick the constant $\delta / \eta^{5 / 2}$ to be arbitrarily small, (47) can be treated as a singularly perturbed problem with a slow variable $x_{2}$, a fast variable $z_{2}$ and a singular parameter $\varepsilon_{2}$. The critical manifold is an S shaped surface given by the formula $x_{2}=-z_{2}^{3}+z_{2}$. The dynamics of the reduced equation and the layer equation are shown in Figure 15. The dynamics of (47) is very well understood (see [10] and the references therein). Let $\Sigma_{2}^{\text {in }}$ and $\Sigma_{2}^{\text {out }}$ be sections defined using formulas (42) and (43), see Figure 15. The trajectories starting at $\Sigma_{2}^{\text {in }}$ follow the upper branch of the slow manifold until they arrive at the fold point $z_{2}=1 / \sqrt{3}$. Subsequently, they jump to the lower branch of the slow manifold and move further along it until they arrive to $\Sigma_{2}^{\text {out }}$. It is a straightforward exercise, left to the reader, to prove that the dynamics of the full system (46) closely follows the dynamics of (47).

Finally, we discuss the relation between the sections $\Sigma_{-}^{\text {out }}$ and $\Sigma_{2}^{\text {in }}$. To describe the transition between these two sections, we need the coordinate change $\kappa_{12}$ from $K_{\text {en }}$ to $K_{-}$and its inverse, $\kappa_{21}=\kappa_{12}^{-1}$. These transformations are given by

$$
\begin{aligned}
& \kappa_{12}: x_{2}=-\left(-y_{1}\right)^{-3 / 2}, \quad r_{2}=\left(-y_{1}\right)^{1 / 2} r_{1}, \quad z_{2}=z_{1}\left(-y_{1}\right)^{-1 / 2}, \quad \varepsilon_{2}=\varepsilon_{1}\left(-y_{1}\right)^{-5 / 2} ; \\
& \kappa_{21}: r_{1}=r_{2}\left(-x_{2}\right)^{1 / 3}, \quad y_{1}=-\left(-x_{2}\right)^{-2 / 3}, \quad z_{1}=z_{2}\left(-x_{2}\right)^{-1 / 3}, \quad \varepsilon_{1}=\varepsilon_{2}\left(-x_{2}\right)^{-5 / 3} \text {. }
\end{aligned}
$$

It now follows that, if we define

$$
\Sigma_{2}^{\text {in }}=\kappa_{12}\left(\Sigma_{-}^{\text {out }}\right)
$$

then $\tilde{k}=\eta^{-3 / 2}$.

The analysis for the section $K_{+}$is very similar, with the formulas differing by some signs only. The dynamics in $K_{+}$is analogous to the dynamics in $K$ with $\bar{y}_{2}=1$.

\subsection{Dynamics in the exit chart $K_{\mathrm{ex}}$}

In the section, we analyze the dynamics of (31) in the exit chart $K_{\text {ex }}$. Here, the rescalings (32) become

$$
x=r_{1}^{3}, \quad y=r_{1}^{2} y_{1}, \quad z=r_{1} z_{1}, \quad \varepsilon=r_{1}^{5} \varepsilon_{1},
$$


We transform (31) using the variables (48) and apply a time rescaling, cancelling a factor of $r_{1}^{2}$. The resulting system is

$$
\begin{aligned}
& \dot{r}_{1}=\frac{1}{3} \varepsilon_{1} r_{1}\left(1+O\left(r_{1}\right)\right) \\
& \dot{y}_{1}=-\frac{2}{3} \varepsilon_{1} y_{1}+O\left(r_{1} \varepsilon_{1}^{2}\right) \\
& \dot{z}_{1}=-\left(z_{1}^{3}+y_{1} z_{1}+1+\frac{1}{3} \varepsilon_{1} z_{1}\right)+O\left(r_{1}\right) \\
& \dot{\varepsilon}_{1}=-\frac{5}{3} \varepsilon_{1}^{2}\left(1+O\left(r_{1}\right)\right) .
\end{aligned}
$$

As before, $t$ and the overdot are recycled as the independent variable and the derivative with respect to this variable, respectively.

Let $\tilde{\kappa}_{12}$ and $\tilde{\kappa}_{21}$ be chart transitions between $K$ and $K_{\text {ex }}$ analogous to $\kappa_{12}$ and $\kappa_{21}$. In this section we study the transition by the flow between $\Sigma_{\mathrm{ex}}^{\text {in }}=\tilde{\kappa}_{21}\left(\Sigma_{2}^{\text {out }}\right)$ and

$$
\Sigma_{\mathrm{ex}}^{\mathrm{out}} \equiv\left\{\left(r_{1}, y_{1}, z_{1}, \varepsilon_{1}\right): r_{1}=\rho\right\} .
$$

Note that (48) differs only slightly from (34): the only difference is the sign in front of $r_{1}$. Similarly the chart transformations $\tilde{\kappa}_{12}$ and $\tilde{\kappa}_{21}$ differ very little from $\kappa_{12}$ and $\kappa_{21}$, namely $\tilde{\kappa}_{12}$ can be obtained from $\kappa_{12}$ by deleting the $-\operatorname{sign}$ before $\varepsilon_{1}^{-3 / 5}$ and $\tilde{\kappa}_{21}$ can be obtained from $\kappa_{21}$ by deleting the - sign before the terms $z_{2} x_{2}^{-1 / 3}, x_{2}^{-5 / 3}$ and $r_{2} x_{2}^{1 / 3}$. It is easy to check that $\Sigma_{\mathrm{ex}}^{\mathrm{in}}$ is alternatively defined by

$$
\Sigma_{\mathrm{ex}}^{\mathrm{in}} \equiv\left\{\left(r_{1}, y_{1}, z_{1}, \varepsilon_{1}\right): \varepsilon_{1}=\delta\right\} .
$$

System (49) has analogous properties as (35): the hyperplanes $\left\{r_{1}=0\right\}$ and $\left\{\varepsilon_{1}=0\right\}$ and the 2D space $\left\{r_{1}=\varepsilon_{1}=0\right\}$ are also invariant. System (49) restricted to the space $\left\{r_{1}=\varepsilon_{1}=0\right\}$ becomes:

$$
\begin{aligned}
& \dot{y}_{1}=0 \\
& \dot{z}_{1}=-\left(z_{1}^{3}+y_{1} z_{1}+1\right) .
\end{aligned}
$$

There are two curves of equilibria determined by the equation $z_{1}^{3}+y_{1} z_{1}+1=0$. The phase portrait is as follows. Any initial condition starting in $\Sigma_{\mathrm{ex}}^{\mathrm{in}}$ arrives in $\Sigma_{\mathrm{ex}}^{\mathrm{out}}$ and the trajectories are attracted to a codimension one manifold close to the left branch of the curve $z_{1}^{3}+y_{1} z_{1}+1=0$, see Figure 16 .

\subsection{Proof of Theorem 2}

(i) We define transition maps for the flow in the charts and write $\Pi: \Sigma^{\text {in }} \rightarrow \Sigma^{\text {out }}$ as a composition of such maps. The sequence of transition maps that is needed depends on the initial conditions. We will consider one such choice in some detail and outline the proof for the other cases. Let $\Pi_{\mathrm{in} \rightarrow 2}: \Sigma_{\mathrm{en}}^{\mathrm{in}} \rightarrow \Sigma_{2}^{\mathrm{in}}$ be the composition of the blow up restricted to $\Sigma^{\text {in }}$, the transition map from $\Sigma_{\mathrm{en}}^{\text {in }}$ to $\Sigma_{\mathrm{en}}^{\text {out }}$ and the chart transformation $\kappa_{12}$. Let $\Pi_{2}: \Sigma_{2}^{\text {in }} \rightarrow \Sigma_{2}^{\text {out }}$ be the transition map in $K$. Let $\Pi_{2 \rightarrow \text { out }}: \Sigma_{2}^{\text {out }} \rightarrow \Sigma_{\text {ex }}^{\text {out }}$ be the composition of the chart transformation $\tilde{\kappa}_{21}$ with the map from $\Sigma_{\text {ex }}^{\text {in }}$ to $\Sigma_{\text {ex }}^{\text {out }}$ and the 'blow down' restricted to $\Sigma_{\mathrm{ex}}^{\text {out }}$. The intermediate maps $\Pi_{\mathrm{in} \rightarrow 2}, \Pi_{2}$ and $\Pi_{2 \rightarrow \text { out }}$ are smooth. The arguments in Section 4.1 imply that $\Pi=\Pi_{\text {in } \rightarrow 2} \circ \Pi_{2} \circ \Pi_{2 \rightarrow \text { out }}$ for initial conditions satisfying $y_{0}=O\left(\varepsilon^{2 / 5}\right)$. The domain of $\Pi_{\text {in } \rightarrow 2} \circ \Pi_{2} \circ \Pi_{2 \rightarrow \text { out }}$ is an open set in $\Sigma^{\text {in }}$ obtained as an intersection of a neighborhood of the cusp with $\Sigma^{\text {in }}$ and with the set defined by the condition $\left|y_{0}\right|<K \varepsilon^{2 / 5}$, for $K>0$ an arbitrary but fixed constant. Combining the information in Sections 4.1, 4.2 and 4.4 we conclude that $\Pi_{\mathrm{in} \rightarrow 2} \circ \Pi_{2} \circ \Pi_{2 \rightarrow \text { out }}$ is a smooth diffeomorphism on its domain and its image is contained in a small neighborhood of the cusp intersected with $\Sigma^{\text {out }}$.

Similarly we define maps $\Pi_{\mathrm{in} \rightarrow+}, \Pi_{+}, \Pi_{+\rightarrow \text { out }}, \Pi_{\mathrm{in} \rightarrow-}, \Pi_{-}$and $\Pi_{-\rightarrow \mathrm{out}}$. Arguing as in the case of $\Pi_{\mathrm{in} \rightarrow 2} \circ$ $\Pi_{2} \circ \Pi_{2 \rightarrow \text { out }}$ we conclude that $\Pi_{\mathrm{in} \rightarrow+} \circ \Pi_{+} \circ \Pi_{+\rightarrow \text { out }}$ and $\Pi_{\mathrm{in} \rightarrow-} \circ \Pi_{-} \circ \Pi_{-\rightarrow \text { out }}$ are smooth diffeomorphisms on their domains and their images are contained in a small neighborhood of the cusp with $\Sigma^{\text {out }}$. Moreover, it follows from the analysis in Sections $4.1-4.4$ that the domains of the three composite maps can be chosen so that they overlap and their union is an intersection of a neighborhood of the cusp intersected with $\Sigma^{\text {in }}$. 


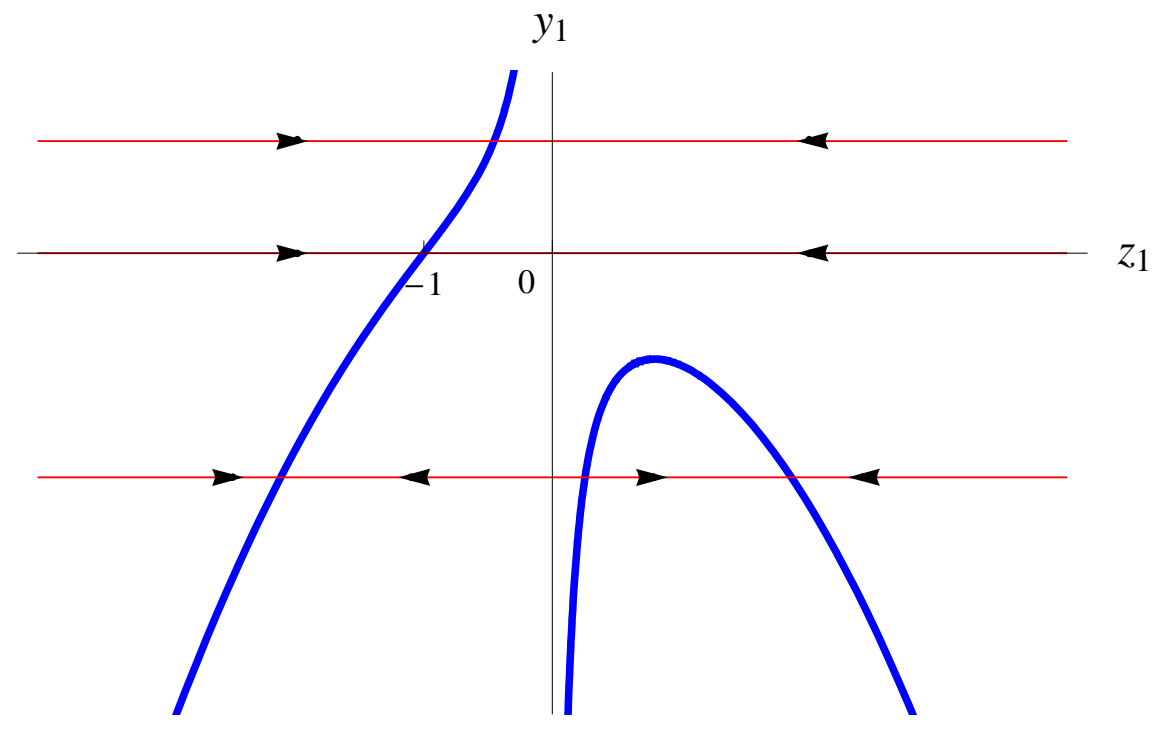

Figure 16: Dynamics in chart $K_{\mathrm{ex}}$.

Item (i) has thus been proved.

(ii) The manifold $S_{\varepsilon}^{+}$is not unique. It can be defined uniquely by specifying its boundary in $\Sigma^{\text {out }}$. This boundary can be taken to be $\Pi\left(S_{\varepsilon}^{-} \cap \Sigma^{\text {in }}\right)$.

(iii) We consider the case when $\Pi$ is given by $\Pi_{\mathrm{in} \rightarrow 2} \circ \Pi_{2} \circ \Pi_{2 \rightarrow \text { out }}$ and leave the other cases to the reader. Note that $\Pi_{2}$ is neutral in all directions while $\Pi_{\text {in } \rightarrow 2}$ and $\Pi_{2 \rightarrow \text { out }}$ are exponentially contracting in the $z_{1}$ direction and neutral in the $y_{1}$ direction (see Figures 13 and 16).

\section{Analysis for initial conditions lying below a neighborhood of the cusp point}

Here, we study orbits through initial conditions that lie below a neighborhood of the cusp point. To that end, we define a new section

$$
\Sigma_{\mathrm{bot}}=\left\{(x, y, z, \varepsilon): z=-\rho, 0 \leq \varepsilon \leq \varepsilon_{0}\right\},
$$

where $\rho>0$ and $\varepsilon_{0}$ are positive and small. The initial conditions we study lie on $\Sigma_{\text {bot }}$, and to study the orbits through these initial conditions it is useful to include higher order terms in the vector field (31),

$$
\begin{aligned}
& x^{\prime}=\varepsilon(1+O(x, y, z, \varepsilon)) \\
& y^{\prime}=\varepsilon\left(C z+O\left(x, y, z^{2}, \varepsilon\right)\right) \\
& z^{\prime}=-\left(z^{3}+y z+x\right)+O\left(x z, y z^{3}, z^{4}, \varepsilon\right) .
\end{aligned}
$$

\subsection{The main result of this section}

The main result of this section is:

Proposition 3 On the section $\Sigma_{\mathrm{bot}}$, there exists a sufficiently small rectangle $R_{\mathrm{bot}}$, which contains the point of intersection of $\Sigma_{\mathrm{bot}}$ with the critical fiber of the cusp point, such that the following statements hold:

1. The rectangle $R_{\mathrm{bot}}$ is mapped onto an exponentially thin strip about $S_{\varepsilon}^{+} \cap \Sigma^{\mathrm{out}}$,

2. There exists a strip contained in $R_{\mathrm{bot}}$ on which $D \Pi$ is exponentially large.

The proof of this proposition is given below. As a preliminary step, we introduce one other useful chart. 


\subsection{Chart $K_{b o t}$}

In order to prove Proposition 3, we introduce a new chart in which we may study the approach to the cusp point along the critical fiber and the nearby dynamics. This chart corresponds to setting $\bar{z}=-1$ in (32).

$$
x=r_{b}^{3} x_{b}, \quad y=r_{b}^{2} y_{b}, \quad z=-r_{b}, \quad \varepsilon=r_{b}^{5} \varepsilon_{b} .
$$

In chart $K_{b o t}$, system (51) becomes (after a time rescaling and with ' denoting the derivative with respect to the new time variable):

$$
\begin{aligned}
& x_{b}^{\prime}=\varepsilon_{b}+3 x_{b} F_{b}\left(x_{b}, y_{b}, r_{b}, \varepsilon_{b}\right)+O\left(r_{b}\right) \\
& y_{b}^{\prime}=2 y_{b} F_{b}\left(x_{b}, y_{b}, r_{b}, \varepsilon_{b}\right)+O\left(r_{b}\right) \\
& r_{b}^{\prime}=-r_{b} F_{b}\left(x_{b}, y_{b}, r_{b}, \varepsilon_{b}\right) \\
& \varepsilon_{b}^{\prime}=5 \varepsilon_{b} F_{b}\left(x_{b}, y_{b}, r_{b}, \varepsilon_{b}\right)
\end{aligned}
$$

with $F_{b}\left(x_{b}, y_{b}, r_{b}, \varepsilon_{b}\right)=1+y_{b}-x_{b}+O\left(r_{b}\right)$.

Next, we recall the blow-up in chart $K_{-}$. The chart-to-chart transformation from $K_{\text {bot }}$ to $K_{-}$is given as follows:

$$
x_{2}=\left(-y_{b}\right)^{-3 / 2} x_{b}, \quad r_{2}=\left(-y_{b}\right)^{1 / 2} r_{b}, \quad z_{2}=-\left(-y_{b}\right)^{-1 / 2}, \quad \varepsilon_{2}=\left(-y_{b}\right)^{-5 / 2} \varepsilon_{b} .
$$

We are interested in trajectories which ultimately cross the section $\Sigma_{2, \text { bot }}$, defined by $z_{2}=-L$, where $L$ is some large number. Under the coordinate change between charts $K_{\text {bot }}$ and $K_{-}$, this section corresponds to a section in $K_{\text {bot }}$ defined by $y_{b}=-\frac{1}{L^{2}}$. Denote this section by $\Sigma_{\text {bot }}^{\text {out }}$. We also introduce the section $\Sigma_{\text {bot }}^{\text {in }}$ defined by $r_{b}=\rho$. It corresponds to $\Sigma_{\text {bot }}$ in chart $K_{\text {bot }}$. Now, by choosing the rectangle $R_{\text {bot }}$ such that it is sufficiently small then we know that the function $F_{b}$ is bounded away from zero and that the transition from $\Sigma_{\text {bot }}^{\text {in }}$ to $\Sigma_{\text {bot }}^{\text {out }}$ is a regular smooth passage near the saddle point $\left(x_{b}, y_{b}, r_{b}, \varepsilon_{b}\right)=(0,0,0,0)$. Hence, with the suitable choice of $R_{\text {bot }}$, its image in $\Sigma_{2}$,bot under the coordinate change contains the set defined by the conditions $r_{2}=0, x_{2}=-1 /(2 \sqrt{3})$ (and $\varepsilon_{2}$ sufficiently small). This basic dynamics is central to the proof of the proposition, as we now show.

\subsection{Proof of Proposition 3}

We first prove part 1 of the proposition. Let $\Sigma_{2 \text {,int }}$ be defined by $x_{2}=1 /(2 \sqrt{3})+\delta$, where $\delta>0$ is small. Note that the image of $R_{\mathrm{bot}}$ in $\Sigma_{2 \text {,int }}$ is bounded in the $z_{2}$ direction. The maximal distance between different points in the image is approximately equal to the distance between the two folds of system (47), namely $1 / \sqrt{3}$. Finally, and most importantly, as the orbits travel from $\Sigma_{2}$,int to $\Sigma_{2}^{\text {out }}$, there is exponential contraction in the $z_{2}$ direction. Hence, because the time of flight between these two sections is long, part 1 of the proposition holds.

To prove part 2, we include more detail about the higher order terms,

$$
\begin{aligned}
x_{2}^{\prime} & =\varepsilon_{2}\left(1+O\left(r_{2}\right)\right)+\frac{3}{2} r_{2} x_{2} \varepsilon_{2} O\left(r_{2}\right) \\
r_{2}^{\prime} & =-\frac{1}{2} r_{2}^{3} \varepsilon_{2}\left(C z_{2}+O\left(r_{2}\right)\right) \\
z_{2}^{\prime} & =-\left(z_{2}^{3}-z_{2}+x_{2}\right)+\frac{1}{2} r_{2} z_{2} \varepsilon_{2} O\left(r_{2}\right) \\
\varepsilon_{2}^{\prime} & =\frac{5}{2} r_{2}^{2} \varepsilon_{2}^{2}\left(C z_{2}+O\left(r_{2}\right)\right)
\end{aligned}
$$

Recall that system (55) for $r_{2}=0$ corresponds to a singularly perturbed system in two dimensions with parameter $\varepsilon_{2}$ and with S-shaped critical manifold), see Figure 17. The analysis we present is local to the critical fiber $r_{2}=\varepsilon_{2}=0, x_{2}=-1 /(2 \sqrt{3})$. As mentioned, this critical fiber and its neighborhood (one-sided in $r_{2}$ and $\varepsilon_{2}$ ) are contained in the image of $R_{\text {bot }}$ in $\Sigma_{2 \text {,bot }}$. By Fenichel theory, there exist three dimensional manifolds $S_{a, 1}, S_{r}$ and $S_{a, 2}$, see Figure 17. These manifolds exist away from the upper and lower fold surfaces $\mathcal{F}_{u}$ and $\mathcal{F}_{l}$, defined by $\left(x_{2}, y_{2}\right)=(1 / \sqrt{3}, 1 /(2 \sqrt{3}))$ and $\left(x_{2}, y_{2}\right)=(-1 / \sqrt{3},-1 /(2 \sqrt{3}))$, respectively, and they are close to the fold points in the plane $r_{2}=\varepsilon_{2}=0$. 


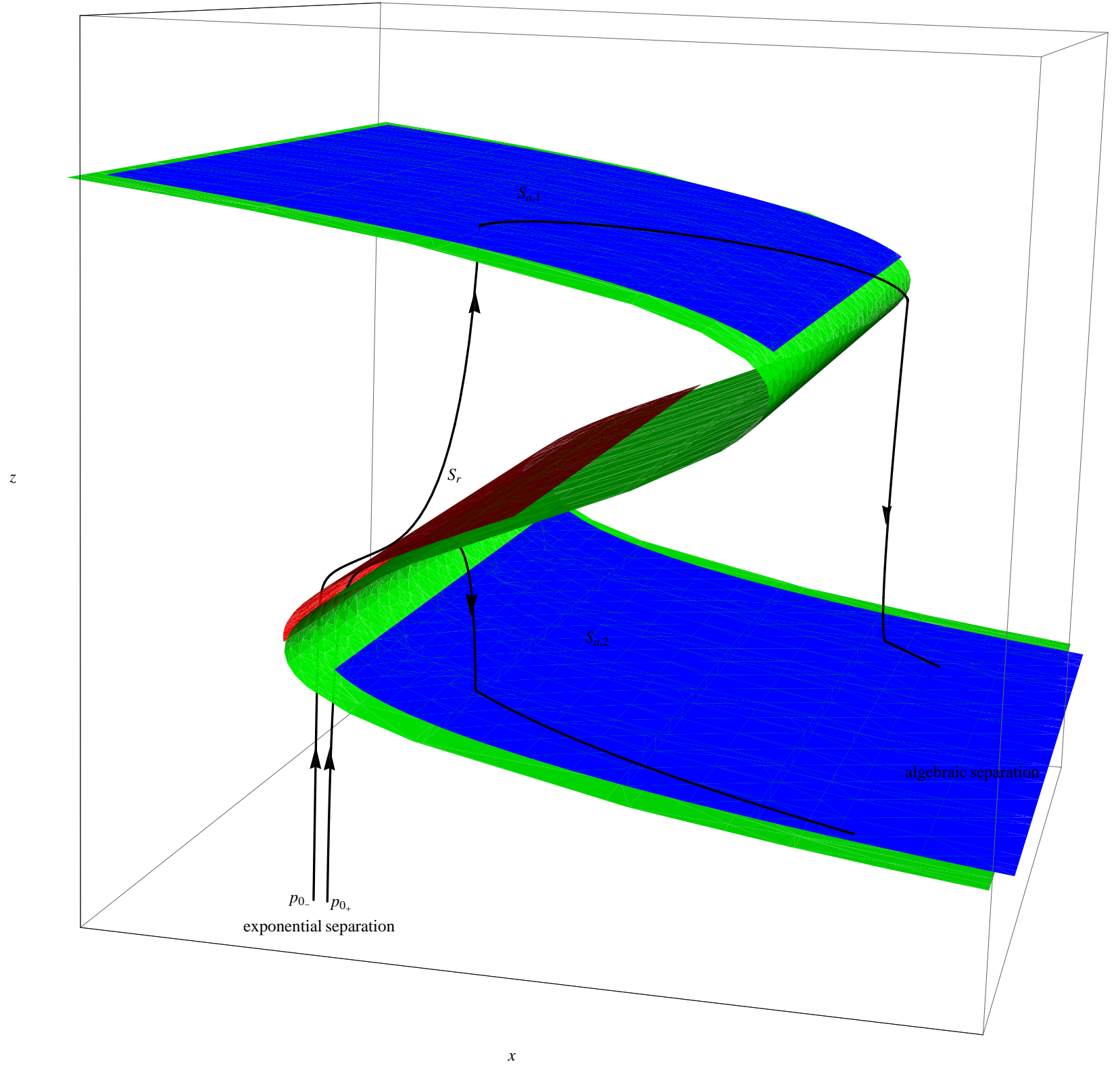

Figure 17: Trajectories $p_{-}(t)$ and $\left.p_{+}(t)\right)$ in the coordinate system $\left(x_{2}, r_{2}, z_{2}\right)$ with $\varepsilon_{2}$ suppressed. 
To prove part 2 of the proposition, we consider two trajectories starting in $\Sigma_{2 \text {,bot }}$, namely a trajectory $p_{-}(t)$, with initial condition $\left(x_{2,-}, r_{2}, \varepsilon_{2},-L\right)$ and a trajectory $p_{+}(t)$, with initial condition $\left(x_{2,+}, r_{2}, \varepsilon_{2},-L\right)$. We pick $p_{-}(t)$ so that it passes close to $S_{r}$, follows it for a time $T=c / \varepsilon_{2}$, where $c$ is a small constant, and then moves up to $S_{a, 1}$. We pick $p_{+}(t)$ so that it passes close to $S_{r}$, follows it for time $T$ and then moves up to $S_{a, 2}$. The trajectories $p_{+}$and $p_{-}$are shown in Figure 17. Note that, due to the long passage time near $S_{r}$, the distance between $p_{+}(0)$ and $p_{-}(0)$ must be exponentially small.

Let $p_{-}^{*}$ (respectively $p_{+}^{*}$ ) be the intersection point of the trajectory $p_{-}(t)$ (respectively $p_{+}(t)$ ) with $\Sigma_{2, \text { int }}$. We claim that the distance between $p_{-}^{*}$ and $p_{+}^{*}$ is algebraic. To see this, we note that the evolution of the $r_{2}$ coordinate along both of the solutions is governed by the term $-\frac{1}{2} r_{2}^{3} \varepsilon_{2} C z_{2}$, recall the $r_{2}^{\prime}$ equation in (55). Note also that during its passage from $\Sigma_{2, \text { bot }}$ to $\Sigma_{2 \text {,int }}$, the trajectory $p_{-}(t)$ (respectively $p_{+}(t)$ ) spends the longest time moving along $S_{a, 1}$ (respectively $S_{a, 2}$ ). Moreover, during this passage, the term $-\frac{1}{2} r_{2}^{3} \varepsilon_{2} C z_{2}$ is of approximately equal magnitude but of opposite sign for the two trajectories.

The claim now follows. The exponentially small interval between $p_{-}(0)$ and $p_{+}(0)$ is expanded to an interval of $O(\varepsilon)$. Therefore, somewhere in this interval there must be a point $p$ such that $\left|D \Pi_{p}\right|$ is exponentially large.

\section{Application to Zeeman's examples}

\subsection{The results for the heartbeat model (4) with $\varepsilon>0$}

Let $S_{a,+}=\left\{(x, y) \in S_{0}: y>1 / \sqrt{3}\right\}$ and $S_{a,-}=\left\{(x, y) \in S_{0}: y<-1 / \sqrt{3}\right\}$.

Theorem 3 (Excitable system) For sufficiently small $\varepsilon>0$ system (4) is excitable, that is, it has the following properties:

(i) There exists a unique stable equilibrium $p_{a}$, which eventually attracts all the trajectories.

(ii) There exists an open set $V$ such that every trajectory starting in $V$ is attracted to a slow manifold near $S_{a-}$, passes near the fold $\left(-\frac{2}{3 \sqrt{3}}, \frac{1}{\sqrt{3}}\right)$ and is attracted to a slow manifold near $S_{a_{+}}$. The distance from the stable equilibrium to $V$ (the threshold) is equal to $\frac{2}{3 \sqrt{3}}-y_{0}+y_{0}^{3}+o(1)$.

Proof Consider a trajectory with initial condition $(x, y)$ satisfying $x>\frac{2}{3 \sqrt{3}}+\delta$, where $\delta>0$ is a sufficiently small number. Then for $\varepsilon$ sufficiently small this trajectory is attracted to a Fenichel slow manifold $S_{\varepsilon}^{-}$, close to $S_{a,-}$ and must enter a neighborhood of the fold. According to Theorem 6 , this trajectory must exit along the fast fiber and move towards a Fenichel slow manifold $S_{\varepsilon}^{+}$, close to $S_{a,+}$, which contains the stable equilibrium. The threshold is clearly bounded above by $\frac{2}{3 \sqrt{3}}+\delta-x_{0}$, where $x_{0}=y_{0}-y_{0}^{3}$ is the $x$ coordinate of $p_{a}$. The trajectory of $(x, y)$ is shown in Figure 18.

\subsection{The results for the nerve impulse model (7) with $\varepsilon>0$}

Theorem 4 (Smooth return) For equation (7) there exists a slow manifold $S_{\varepsilon}=S_{\varepsilon}^{+} \cup S_{\varepsilon}^{-}$, with $S_{\varepsilon}^{+}$ containing the stable equilibrium and $S_{\varepsilon}^{-}=S_{\varepsilon} \cap\{(x, y, z): z<0\}$. The manifold $S_{\varepsilon}^{+}$is the union of non-empty open sets (in relative topology) $V_{1}, V_{2}, V_{3}, V_{4}$ and $V_{5}$ such that the following statements hold:

(i) $S_{\varepsilon}$ has compact closure and all the trajectories of the system are eventually attracted to it.

(ii) The trajectories starting in $V_{1}$ remain in $S_{+}^{\varepsilon}$ and are attracted to the stable equilibrium.

(iii) The trajectories starting in $V_{2}$ leave $S_{+}^{\varepsilon}$ through the vicinity of the fold line $F_{+}$, are attracted to $S_{\varepsilon}^{-}$, and subsequently follow the slow flow from $S_{\varepsilon}^{-}$to $S_{\varepsilon}^{+}$(smooth return) and are attracted to the stable equilibrium.

(iv) The trajectories starting in $V_{4}$ leave $S_{+}^{\varepsilon}$ through the vicinity of the fold line $F_{+}$, are attracted to $S_{\varepsilon}^{-}$, subsequently leave $S_{\varepsilon}^{-}$through the vicinity of $F_{-}$, are attracted to $S_{\varepsilon}^{+}$(jump return) and finally approach the stable equilibrium. 


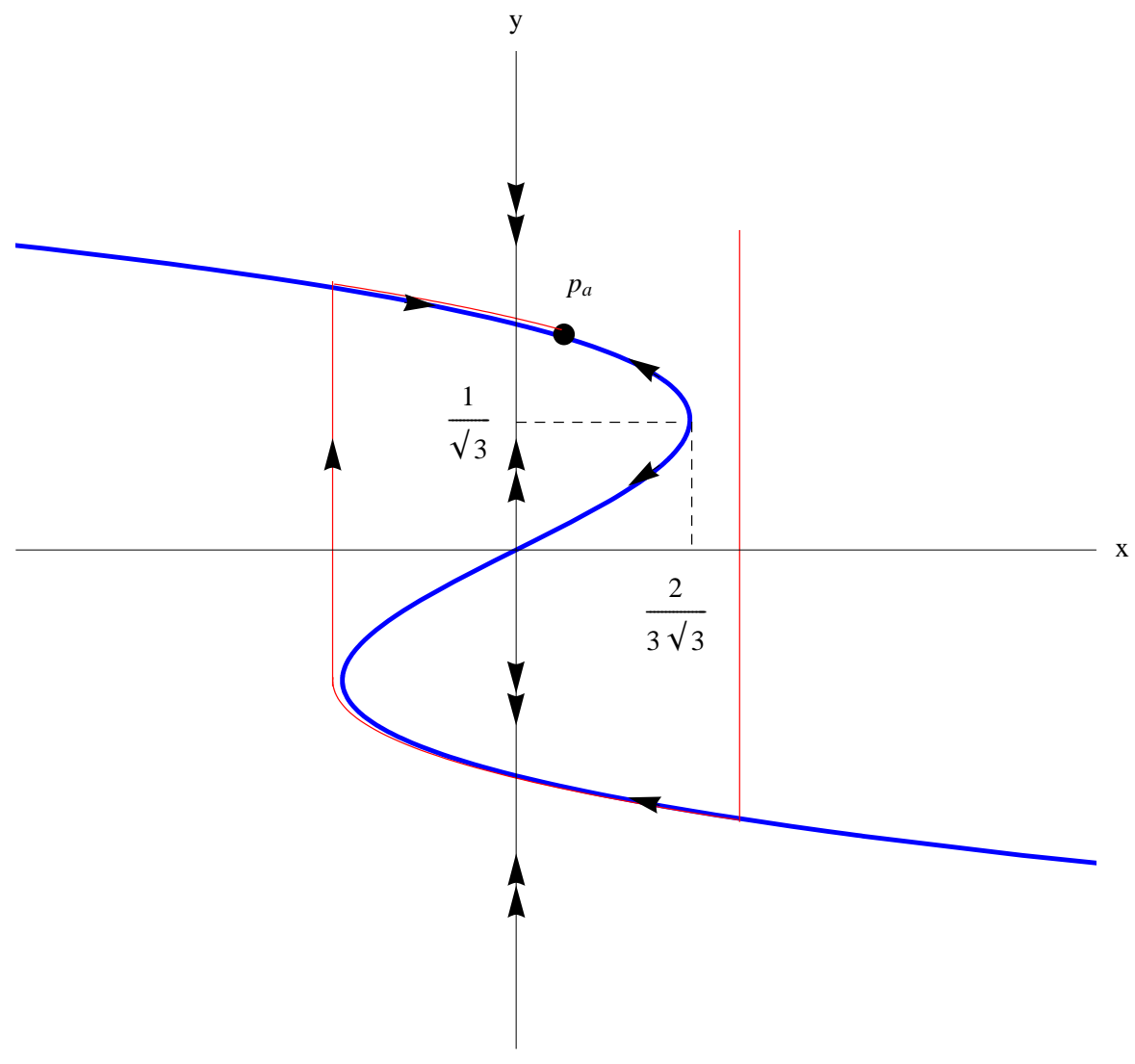

Figure 18: The trajectory of a point $(x, y)$ near the equilibrium $p_{a}$ 
(v) The trajectories starting in $V_{3}$ leave $S_{+}^{\varepsilon}$ through the vicinity of the fold line $F_{+}$, are attracted to $S_{\varepsilon}^{-}$, and subsequently pass from $S_{\varepsilon}^{-}$to $S_{\varepsilon}^{+}$through a small neighborhood of the cusp (transition through cusp) and are attracted to the stable equilibrium.

(vi) The measure of $V_{5}$ is exponentially small (its existence is caused by the canard solution occurring for folded saddle see statement and proof of Theorem 8).

Proof Recall Theorem 1 and the manifolds $S_{0}^{a}, S_{0+}^{a}$ and $S_{0-}^{a}$ (see also Figure 8). It is possible to prove using phase plane methods (Poincaré-Bendixson-like methods) that (19) has a compact absorbing set $R \subset S_{0}^{a}$, containing the point $p_{f}$, such that any singular trajectory eventually enters $R$ and converges to the stable equilibrium. The proof is elementary (but tedious) and will be left to the reader.

We remove a thin strip around $F$ from $R$ and construct a normally hyperbolic (Fenichel) slow manifold approximating the remainder of $R$. This is $S_{\varepsilon}$, with the sets $S_{\varepsilon}^{+}$and $S_{\varepsilon}^{-}$, defined as in the statement of Theorem 4, approximating $S_{0+}^{a} \cap R$ and $S_{0-}^{a} \cap R$. Now Theorems 5, 7, 8 and 2 describe how trajectories travel from $S_{\varepsilon}^{+}$to $S_{\varepsilon}^{-}$and vice-versa. The sets $V_{1}, V_{2}, V_{3}$ and $V_{4}$ are defined by the properties of the trajectories passing through them, for example $V_{2}$ is defined as the set of points whose trajectories leave $S_{+}^{\varepsilon}$ through the vicinity of the fold line $F_{+}$, are attracted to $S_{\varepsilon}^{-}$, and subsequently follow the slow flow from $S_{\varepsilon}^{-}$to $S_{\varepsilon}^{+}$and are attracted to the stable equilibrium. Theorem 1 implies that the sets $V_{3}$ and $V_{4}$ are non-empty. The set $V_{5}$ is defined by all the trajectories entering $U_{2}$ of Theorem 8 .

\section{A Fenichel's theorem - review}

Consider a singularly perturbed equation

$$
\begin{aligned}
& x^{\prime}=\varepsilon f(x, y) \\
& y^{\prime}=g(x, y), \quad x \in \mathbb{R}^{k}, y \in \mathbb{R}^{m}, 0<\varepsilon \ll 1 .
\end{aligned}
$$

Recall the constraining manifold (or critical manifold) $S_{0}$ defined by

$$
S_{0}=\{(x, y) \mid g(x, y)=0\} .
$$

Suppose $\tilde{S}_{0}$ is an open subset of $S_{0}$. Then $\tilde{S}_{0}$ is normally hyperbolic if for every $(x, y) \in \operatorname{cl}\left(\tilde{S}_{0}\right)$ the matrix $D_{y} g$ has no eigenvalues on the imaginary axis.

Theorem 5 (Fenichel)[6, 7] If $\tilde{S}_{0}$ is normally hyperbolic then, for $\varepsilon>0$ and sufficiently small, there exists a locally invariant manifold $S_{\varepsilon}$ close to $\tilde{S}_{0}$ in the $C^{1}$ topology. The manifold $S_{\varepsilon}$ is diffeomorphic to $\tilde{S}_{0}$, and the flow on $S_{\varepsilon}$ is close to the flow of the reduced (constrained) equation on $\tilde{S}_{0}$.

\section{B Folds and folded saddles for $\varepsilon>0$ - review}

\section{B.1 Simple folds}

In this article, we need the description of the dynamics for folds with one fast and one slow variable (for the heartbeat model) and for folds with one fast and two slow variables (for the nerve impulse model). We begin by stating the result for the case of one slow variable and then introduce the theorem for two slow variables as its generalization. The result we state is Theorem 2.1 of [10]. We consider the equation

$$
\begin{aligned}
& x^{\prime}=\varepsilon f(x, y, \varepsilon), \\
& y^{\prime}=g(x, y, \varepsilon), \quad x, y \in \mathbb{R} \\
& \varepsilon^{\prime}=0
\end{aligned}
$$

and assume that

$$
g(0,0,0)=0, \quad \frac{\partial g}{\partial y}(0,0,0)=0
$$




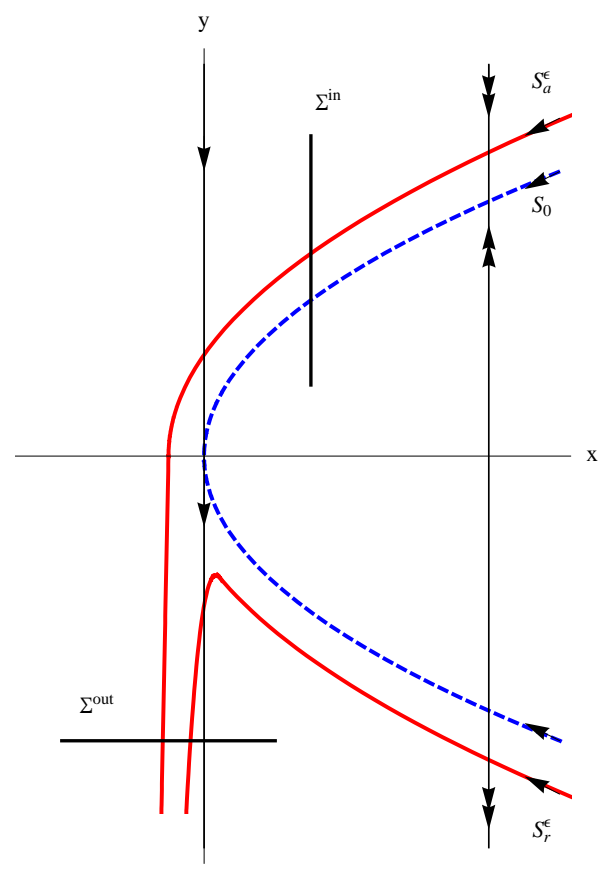

Figure 19: Critical manifold, slow manifolds, and sections for a simple fold

Further, we make non-degeneracy assumptions

$$
\frac{\partial^{2} g}{\partial y^{2}}(0,0,0) \neq 0, \quad \frac{\partial g}{\partial x}(0,0,0) \neq 0, \quad f(0,0,0) \neq 0
$$

In particular, we assume that

$$
\frac{\partial^{2} g}{\partial y^{2}}(0,0,0)>0, \quad \frac{\partial g}{\partial x}(0,0,0)>0, \quad f(0,0,0)<0 .
$$

These assumptions may be made without loss of generality, and the latter assumption determines that the direction of the flow is towards the fold (the fold point is a jump point). The information above is summarized in Figure 19.

We define the following sections of the flow:

$$
\Sigma^{i n}=\left\{\left(\rho^{2}, y\right), y \in R\right\}
$$

and

$$
\Sigma^{\text {out }}=\{(x, \rho), x \in \mathbb{R}\}
$$

Also, we let $\Pi: \Sigma^{i n} \rightarrow \Sigma^{\text {out }}$ be the transition map for the flow of (56).

Theorem 6 (simple fold with one slow variable) [10] For system (56) with assumptions (57) and (58), there exist $\varepsilon_{0}>0$ and a neighborhood $U$ of $(0,0)$ such that the following assertions hold for $\varepsilon \in\left(0, \varepsilon_{0}\right]$ :

1. The manifold $S_{a, \varepsilon}$ passes through $\Sigma^{\text {out }}$ at a point $(h(\varepsilon), \rho)$ where $h(\varepsilon)=O\left(\varepsilon^{2 / 3}\right)$.

2. The transition map $\Pi: \Sigma^{i n} \cap U \rightarrow \Sigma^{o u t}$ is a contraction with contraction rate $O\left(e^{-c / \varepsilon}\right)$, where $c$ is a positive constant. 


\section{B.2 Simple folds in problems with two slow dimensions}

Consider the equation

$$
\begin{aligned}
& x^{\prime}=\varepsilon f_{1}(x, y, z, \varepsilon) \\
& y^{\prime}=\varepsilon f_{2}(x, y, z, \varepsilon) \\
& z^{\prime}=g(x, y, z, \varepsilon), \quad x, y, z \in \mathbb{R}^{1}, 0<\varepsilon \ll 1 .
\end{aligned}
$$

Let $S_{0}$ be the critical manifold, and define the fold line

$$
F=\left\{(x, y, z) \in S_{0} \mid \frac{\partial g}{\partial z}(x, y, z, 0) \neq 0\right\} .
$$

Suppose that $(0,0,0) \in F$ and conditions $(\mathrm{B})$ and $(\mathrm{C})$ are satisfied. Further, we assume without loss of generality that

$$
\frac{\partial^{2} g}{\partial z^{2}}(0,0,0,0)>0, \quad \frac{\partial g}{\partial x}(0,0,0,0)>0
$$

We define the sections of the flow analogously as in the case of a simple fold. Namely, we introduce

$$
\Sigma^{i n}=\left\{(x, y, z) \mid(x, y, z)=\left(\rho^{2}, y, z\right),(y, z) \in \mathbb{R}^{2}\right\}
$$

and

$$
\Sigma^{\text {out }}=\left\{(x, y, z) \mid(x, y, z)=(x, y, \rho),(x, y) \in \mathbb{R}^{2}\right\} .
$$

Theorem 7 (simple folds in problems with two slow variables) [13] For system (59) with the general nondegenracy conditions $(A),(B)$, and $(C)$, as well as with condition (60), there exist $\varepsilon_{0}>0$ and a neighborhood $U$ of $(0,0,0)$ such that the following assertions hold for $\varepsilon \in\left(0, \varepsilon_{0}\right]$ :

(i) The transition map $\Pi: \Sigma^{i n} \cap U \rightarrow \Sigma^{\text {out }}$ induced by the flow of (59) is a diffeomorphism mapping a neighborhood of $S_{0} \cap U$ into $\Sigma^{o u t}$. Any trajectory starting at $S_{a, \varepsilon} \cap \Sigma^{\text {in }}$ passes through $\Sigma^{\text {out }}$ at a point whose distance to the set $\{(x, y, z+\rho) \mid(x, y, z) \in F \cap U\}$ is $O\left(\varepsilon^{2 / 3}\right)$.

(ii) The map $\Pi$ is exponentially contracting in the $z$ direction. More precisely,

$$
\left\|\frac{\partial \Pi}{\partial z}\right\|=O\left(e^{-c / \varepsilon}\right)
$$

where $c$ is a positive constant.

(iii) The contraction/expansion of $\Pi$ in the direction of the fold is uniformly bounded; namely, if $v$ is the tangent vector to the fold at some point $(x, y, z) \in F \cap U$ then there exists a constant $K>0$ such that

$$
\frac{1}{K} \leq|\operatorname{grad} \Pi(x, y, z) \cdot v| \leq K .
$$

Remark 1 In [13], the statement of the fold Theorem (Theorem 1) is preceded by transformation to a "normal form," which has the effect of straightening some of the manifolds. We have chosen to state the theorem without the preliminary transformation. Our result can be obtained by a combination of Theorem 1 of [13] and their result on the preliminary transformation.

We have purposely weakened the statement of item (iii) as the estimate we give is sufficient for the purposes of this article and the statement becomes simpler. We refer the reader to [13] for sharper estimates. 


\section{B.3 Folded saddles}

We use the notation and definition of the sections of the flow as in Section B.2, as well as the hypotheses there, with one difference. Namely, we assume that (B) is violated, that is we assume

$$
f(0,0,0,0) \cdot \operatorname{grad} g(0,0,0,0)=0 .
$$

The defining condition for a folded saddle is stated using the desingularized equation (15) derived in Section 2.3. Note that condition (61) implies that $(0,0)$ is an equilibrium of $(15)$. Then, $(0,0,0,0)$ is a folded saddle and $(0,0)$ is a saddle type equilibrium of $(15)$. Further, a non-degeneracy condition is needed on the function

$$
F_{c}(x, y, z, \varepsilon)=f(x, y, z, \varepsilon) \cdot g(x, y, z, \varepsilon) ;
$$

namely, if $v$ is a tangent vector to the fold line $F$ at $(0,0,0,0)$ then

$$
\operatorname{grad} F_{c}(0,0,0,0) \cdot v \neq 0 .
$$

Note that, for $\rho$ sufficiently small, $\Sigma^{i n}$ is now naturally divided into the following two regions:

$$
\Sigma_{+}^{i n}=\left\{(x, y, z) \in \Sigma^{i n} \mid F_{c}(x, y, z, \varepsilon)>0\right\} \quad \text { and } \quad \Sigma_{-}^{i n}=\left\{(x, y, z) \in \Sigma^{i n} \mid F_{c}(x, y, z, \varepsilon)<0\right\}
$$

Then, we have the following theorem:

Theorem 8 (Folded saddle) [12, 13] For system (59) with the general assumptions (A) and (C), as well as (61) and (62), there exist $\varepsilon_{0}>0$, a neighborhood $U$ of $(0,0,0)$, and open sets $U_{1}, U_{2}$ and $U_{3}$ such that the following assertions hold for $\varepsilon \in\left(0, \varepsilon_{0}\right]$ :

(i) $U=U_{1} \cup U_{2} \cup U_{3}, U_{1} \cap U_{3}=\emptyset, U_{1} \cup U_{2} \subset \Sigma_{+}^{\text {in }}$ and $U_{2}$ is exponentially thin, i.e. there exists $c>0$ such that for every point $p \in U_{2}$ the length of the line segment $\left\{q \in U_{2} q=p+s v\right.$ for some $\left.s \in \mathbb{R}\right\}$ is bounded by $e^{-c / \varepsilon}$.

(ii) The transition map $\Pi: \Sigma^{i n} \cap U_{1} \rightarrow \Sigma^{\text {out }}$ induced by the flow of (59) is a diffeomorphism mapping a neighborhood of $S_{0} \cap U$ into $\Sigma^{o u t}$. Any trajectory starting at $S_{a, \varepsilon} \cap \Sigma^{\text {in }}$ passes through $\Sigma^{\text {out }}$ at a point whose distance to the set $\{(x, y, z+\rho) \mid(x, y, z) \in F \cap U\}$ is $O\left(\varepsilon^{2 / 3}\right)$.

(iii) The map $\Pi$ is exponentially contracting in the $z$ direction. More precisely,

$$
\left\|\frac{\partial \Pi}{\partial z}\right\|=O\left(e^{-\tilde{c} / \varepsilon}\right)
$$

where $\tilde{c}$ is a positive constant.

(iv) The contraction/expansion of $\Pi$ in the direction of the fold is algebraic in $\varepsilon$, namely there exists a constant $\alpha>0$ such that if $v$ is the tangent vector to the fold at some point $(x, y, z) \in F \cap U$ then

$$
\left.\varepsilon^{\alpha} \leq \mid \operatorname{grad} \Pi(x, y, z) \cdot v\right) \mid \leq \frac{1}{\varepsilon^{\alpha}} .
$$

(v) Trajectories starting in $U_{2}$ turn around before reaching $\Sigma^{\text {out }}$ and return to $S_{a, \varepsilon}$.

The sets $U_{1}, U_{2}$ and $U_{3}$ are shown in Figure 20.

Sketch of the proof Theorem 8 (or a similar version) is not stated in either [12] or [13], but it follows closely from the results and arguments given in these articles. In this article, we sketch the proof of the result, referring the reader to [12], [13]. Let $S_{0}$ be the critical manifold for (59) and let

$$
S_{0}^{a}=\left\{(x, y, z) \in S_{0} \mid \frac{\partial g}{\partial z}(x, y, z, 0)<0\right\}
$$

and

$$
S_{0}^{r}=\left\{(x, y, z) \in S_{0} \mid \frac{\partial g}{\partial z}(x, y, z, 0)>0\right\} .
$$




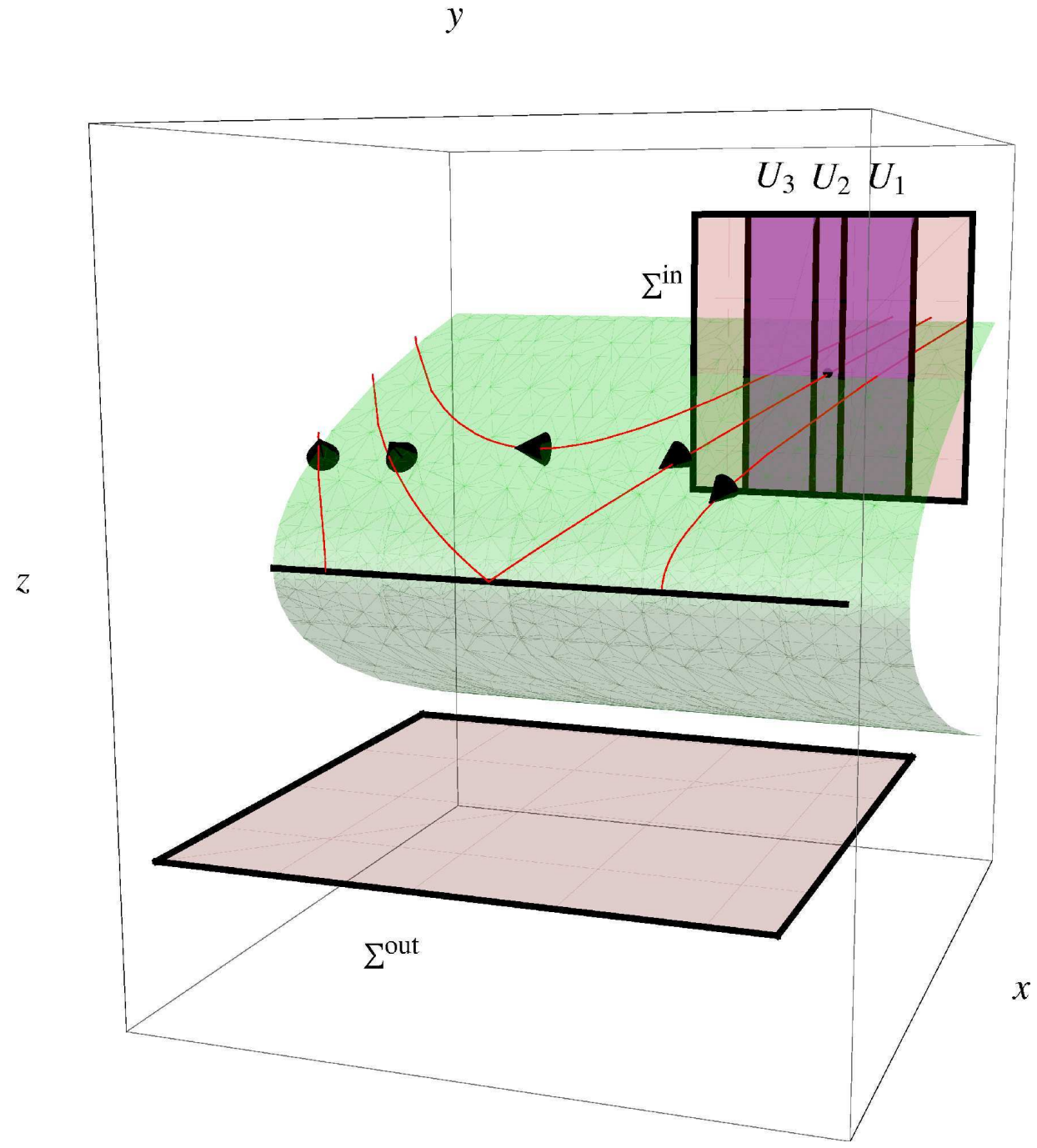

Figure 20: Dynamics in chart $K_{\text {ex }}$. 
Removing a small neighborhood of the fold, we construct normally hyperbolic (Fenichel) slow manifolds $S_{\varepsilon}^{a}$ and $S_{\varepsilon}^{r}$. In [12], it is proved that for a fixed choice of the Fenichel manifolds $S_{\varepsilon}^{a}$ and $S_{\varepsilon}^{r}$ there exists a unique canard solution, i.e. a solution connecting from $S_{\varepsilon}^{a}$ to $S_{\varepsilon}^{r}$. The canard solution divides $S_{\varepsilon}^{a}$ into regions with two types of dynamics: on one side solutions pass to $\Sigma^{\text {out }}$ and continue on along the fast direction and on the other side solutions return to $S_{\varepsilon}^{a}$. These regions correspond to $U_{1}$ and $U_{3}$. In between there is an exponentially thin region centered at the canard solution consisting of trajectories that follow $S_{\varepsilon}^{r}$ in a similar way as in classical canard phenomenon (canard explosion), see for example [11]. This region corresponds to $U_{2}$. We note that the flow in the direction of the fold line is more complicated due to the saddle structure, which accounts for the weaker version of statement (iii) in comparison to Theorem 7.

\section{References}

[1] E. Benoit, Systemes lentes-rapides en $\mathbb{R}^{3}$ et leur canards, Asterisque 109-110, 159-191 (1983).

[2] F. Dumortier [1993], Techniques in the theory of local bifurcations: blow-up, normal forms, nilpotent bifurcations, singular perturbations, in Bifurcations and Periodic Orbits of Vector Fields, NATO ASI Series C, Mathematical and Physical Sciences, 408 , 19-73, D. Schlomiuk ed., Dordrecht: Kluwer.

[3] F. Dumortier and R. Roussarie [1996], Canard cycles and center manifolds, Mem. Am. Math. Soc. 121 (577) 1-100.

[4] F. Dumortier and R. Roussarie [2001], Geometric singular perturbation theory beyond normal hyperbolicity, in Multiple-Time-Scale Dynamical Systems, IMA Vol. Math. Appl. 122, 29-63, C. K. R. T. Jones and A. Khibnik, eds., New York: Springer.

[5] F. Dumortier, R. Roussarie, and J. Sotomayor [1997], Bifurcations of cuspidal loops, Nonlinearity 10 $1369-1408$.

[6] N. Fenichel, Geometric singular perturbation theory, JDE 31, 53-98, 1979.

[7] M.W. Hirsch, C.C. Pugh, M. Shub, Invariant Manifolds, LNM 583, Springer-Verlag, Berlin, 1977.

[8] C. K. R. T. Jones, Geometric singular perturbation theory, in Dynamical Systems LNM 1609, 44-120, Springer-Verlag, Berlin, 1995.

[9] J. Keener and J. Sneyd, Mathematical Physiology, Interdisciplinary Applied Mathematics, 8, SpringerVerlag, New York, 1998.

[10] M. Krupa and P. Szmolyan, Extending geometric singular perturbation theory to nonhyperbolic points: fold and canard points in two dimensions, SIAM J. Math. Anal. 33, 286-314, 2001.

[11] M. Krupa and P. Szmolyan, Relaxation oscillation and canard explosion, J. Diff. Eqns. 174, 312-368, 2001.

[12] P. Szmolyan and M. Wechselberger, Canards in $\mathbb{R}^{3}, J D E, \mathbf{1 7 7 ( 2 )}$, 419-453, 2001.

[13] P. Szmolyan and M. Wechselberger, Relaxation oscillations in $\mathbb{R}^{3}, J D E, \mathbf{2 0 0}, 69-104,2004$.

[14] F. Takens, Constrained equations: a study of implicit differential equations and their discontinuous solutions, in Structural stability, the theory of catastrophes, and applications in the sciences, LNM 525, 134-234, Springer-Verlag, Berlin, 1976.

[15] H. Rotstein, M. Wechselberger, and N. Kopell, Canard Induced Mixed-Mode oscillations in a Medial Entorhinal Cortex Layer II Stellate Cell Model, SIADS submitted.

[16] R. Thom, Ensembles et Morphismes Stratifies, B.A.M.S. 75, 240-284, 1969.

[17] R. Thom, L'evolution temporelle de catastrophes, in Applications of global analysis I, Rijksuniversiteit Utrecht. Year? 
[18] R. Thom, Structural Stability and Morphogenesis. An Outline of a General Theory of Models, Second Edition, Addison-Wesley, Redwood City, CA (1989) (English; French original).

[19] E.C. Zeeman, Differential Equations for the heartbeat and nerve impulse. In Towards a Theoretical Biology Edinburgh University Press, Vol. 4, 1972, p. 8-67. 